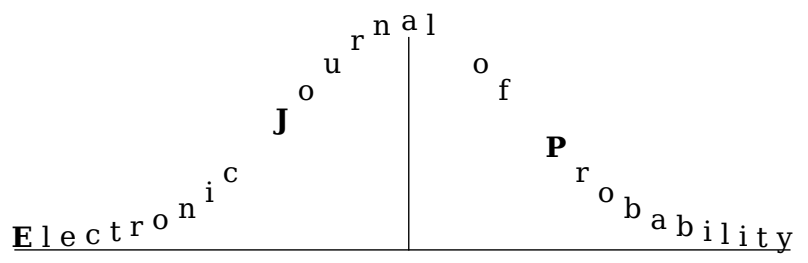

Electron. J. Probab. 25 (2020), article no. 112, 1-23.

ISSN: 1083-6489 https://doi.org/10.1214/20-EJP504

\title{
A type of globally solvable BSDEs with triangularly quadratic generators*
}

\author{
Peng Luo ${ }^{\dagger}$
}

\begin{abstract}
The present paper is devoted to the study of the well-posedness of a type of BSDEs with triangularly quadratic generators. This work is motivated by the recent results obtained by Hu and Tang [14] and Xing and Žitković [28]. By the contraction mapping argument, we first prove that this type of triangularly quadratic BSDEs admits a unique local solution on a small time interval whenever the terminal value is bounded. Under additional assumptions, we build the global solution on the whole time interval by stitching local solutions. Finally, we give solvability results when the generators have path dependence in value process.
\end{abstract}

Keywords: BSDEs; triangularly quadratic generators; BMO martingales; path dependence. MSC2020 subject classifications: $60 \mathrm{H} 10 ; 60 \mathrm{H} 30$.

Submitted to EJP on February 29, 2020, final version accepted on July 26, 2020.

\section{Introduction}

Backward stochastic differential equations (BSDEs) are introduced in Bismut [1]. A BSDE is an equation of the form

$$
Y_{t}=\xi+\int_{t}^{T} g\left(s, Y_{s}, Z_{s}\right) d s-\int_{t}^{T} Z_{s} d W_{s}, \quad t \in[0, T],
$$

where $W$ is a $d$-dimensional Brownian motion, the terminal condition $\xi$ is an $n$-dimensional random variable, and $g: \Omega \times[0, T] \times \mathbb{R}^{n} \times \mathbb{R}^{n \times d} \rightarrow \mathbb{R}^{n}$ is the generator. A solution consists of a pair of predictable processes $(Y, Z)$ with values in $\mathbb{R}^{n}$ and $\mathbb{R}^{n \times d}$, called the value and control process, respectively. The first existence and uniqueness result for BSDEs with an $L^{2}$-terminal condition and a generator satisfying a Lipschitz growth condition is due to Pardoux and Peng [24]. In case that the generator satisfies a quadratic growth condition in the control $z$, the situation is more involved and a general existence theory

${ }^{*}$ Financial support from the Natural Sciences and Engineering Research Council of Canada, Grant RGPIN2017-04054.

${ }^{\dagger}$ School of Mathematical Sciences, Shanghai Jiao Tong University, China.

E-mail: peng. luo@sjtu.edu.cn 
A type of globally solvable BSDEs with triangularly quadratic generators

does not exist. Frei and dos Reis [12] and Frei [11] provide counterexamples which show that multidimensional quadratic BSDEs may fail to have a global solution. In the one-dimensional case the existence of quadratic BSDE is shown by Kobylanski [19] for bounded terminal conditions, and by Briand and $\mathrm{Hu}[4,5]$ for unbounded terminal conditions. Briand and Elie [3] provide a constructive approach to quadratic BSDEs with and without delay. Solvability results for superquadratic BSDEs are discussed in Delbaen et al. [9], see also Masiero and Richou [23], Richou [25] and Cheridito and Nam [6].

The focus of the present work lies on multidimensional quadratic BSDEs. In case that the terminal condition is small enough the existence and uniqueness of a solution was first shown by Tevzadze [26]. Cheridito and Nam [7] and Hu and Tang [14] obtain local solvability on $[T-\varepsilon, T]$ for some $\varepsilon>0$ of systems of BSDEs with subquadratic generators and diagonally quadratic generators respectively, which under additional assumptions on the generator can be extended to global solutions. Cheridito and Nam [7] provide solvability for Markovian quadratic BSDEs and projectable quadratic BSDEs. Xing and Žitković [28] obtained the global solvability for a large class of multidimensional quadratic BSDEs in the Markovian setting. Frei [11] introduced the notion of split solution and studied the existence of solution for multidimensional quadratic BSDEs by considering a special kind of terminal condition. Jamneshan et al. [16] provide solutions for multidimensional quadratic BSDEs with separated generators. Using a stability approach, Harter and Richou [13] establish an existence and uniqueness result for a class of multidimensional quadratic BSDEs. In Bahlali et al. [2] existence is shown when the generator $g(s, y, z)$ is strictly subquadratic in $z$ and satisfies some monotonicity condition. Multidimensional quadratic BSDEs appear in many applications, such as market making problems (see Kramkov and Pulido [20]), nonzero-sum risk-sensitive stochastic differential games (see El Karoui and Hamadène [10], Hu and Tang [14]) and non-zero sum differential games of BSDEs (see Hu and Tang [15]).

Our results are motivated by the recent works of Hu and Tang [14] and Xing and Žitković [28]. We focus on the solvability of a type of BSDEs with triangularly quadratic generators. More precisely, we study the coupled system of quadratic BSDEs

$$
\left\{\begin{aligned}
Y_{t}^{1}= & \xi^{1}+\int_{t}^{T}\left[\frac{1}{2}\left|Z_{s}^{1}\right|^{2}+Z_{s}^{1} l^{1}\left(s, Y_{s}\right)+h^{1}\left(s, Y_{s}, Z_{s}\right)\right] d s-\int_{t}^{T} Z_{s}^{1} d W_{s}, \\
Y_{t}^{i}= & \xi^{i}+\int_{t}^{T}\left[\frac{1}{2}\left|Z_{s}^{i}\right|^{2}+Z_{s}^{i} l^{i}\left(s, Y_{s}, Z_{s}\right)-k^{i}\left(s, Z_{s}\right)+h^{i}\left(s, Y_{s}, Z_{s}\right)\right] d s \\
& -\int_{t}^{T} Z_{s}^{i} d W_{s}, \quad i=2, \ldots, n .
\end{aligned}\right.
$$

By borrowing some techniques from Hu and Tang [14], we first prove that this type of triangularly quadratic BSDEs admits a unique local solution on a small time interval whenever the terminal value is bounded using a contraction mapping argument. Under additional assumptions, we show that the value process is uniformly bounded. Therefore we build the global solution on the whole time interval by stitching local solutions. Finally, we give solvability results when the generators have path dependence in value process.

The paper is organized as follows. In Section 2, we state the setting and main results. The proofs of our main results are presented in Section 3. We further investigate solvability results for a type of quadratic BSDEs with path dependence in value process in Section 4.

\section{Preliminaries and statement of main results}

Let $W=\left(W_{t}\right)_{t \geq 0}$ be a $d$-dimensional Brownian motion on a probability space $(\Omega, \mathcal{F}, P)$. Let $\left(\mathcal{F}_{t}\right)_{t \geq 0}$ be the augmented filtration generated by $W$. Throughout, we fix a $T \in$ $(0, \infty)$. We endow $\Omega \times[0, T]$ with the predictable $\sigma$-algebra $\mathcal{P}$ and $\mathbb{R}^{n}$ with its Borel $\sigma$-algebra $\mathcal{B}\left(\mathbb{R}^{n}\right)$. Equalities and inequalities between random variables and processes 
A type of globally solvable BSDEs with triangularly quadratic generators

are understood in the $P$-a.s. and $P \otimes d t$-a.e. sense, respectively. The Euclidean norm is denoted by $|\cdot|$ and $\|\cdot\|_{\infty}$ denotes the $L^{\infty}$-norm. $\mathcal{C}_{T}\left(\mathbb{R}^{n}\right)$ denotes the set $C\left([0, T] ; \mathbb{R}^{n}\right)$ of continuous functions from $[0, T]$ to $\mathbb{R}^{n}$. For $p>1$, we denote by

- $\mathcal{S}^{p}\left(\mathbb{R}^{n}\right)$ the set of $n$-dimensional continuous adapted processes $Y$ on $[0, T]$ such that

$$
\|Y\|_{\mathcal{S}^{p}}:=E\left[\sup _{0 \leq t \leq T}\left|Y_{t}\right|^{p}\right]^{\frac{1}{p}}<\infty
$$

- $\mathcal{S}^{\infty}\left(\mathbb{R}^{n}\right)$ the set of $n$-dimensional continuous adapted processes $Y$ on $[0, T]$ such that

$$
\|Y\|_{\mathcal{S} \infty}:=\left\|\sup _{0 \leq t \leq T}\left|Y_{t}\right|\right\|_{\infty}<\infty
$$

- $\mathcal{H}^{p}\left(\mathbb{R}^{n \times d}\right)$ the set of predictable $\mathbb{R}^{n \times d}$-valued processes $Z$ such that

$$
\|Z\|_{\mathcal{H}^{p}}=E\left[\left(\int_{0}^{T}\left|Z_{s}\right|^{2} d s\right)^{\frac{p}{2}}\right]^{\frac{1}{p}}<\infty .
$$

Let $\mathcal{T}$ be the set of all stopping times with values in $[0, T]$. For any uniformly integrable martingale $M$ with $M_{0}=0$ and for $p \geq 1$, we set

$$
\|M\|_{B M O_{p}(P)}:=\sup _{\tau \in \mathcal{T}}\left\|E\left[\left|M_{T}-M_{\tau}\right|^{p} \mid \mathcal{F}_{\tau}\right]^{\frac{1}{p}}\right\|_{\infty} .
$$

The class $\left\{M:\|M\|_{B M O_{p}}<\infty\right\}$ is denoted by $B M O_{p}$, which is written as $B M O(P)$ when it is necessary to indicate the underlying probability measure $P$. In particular, we will denote it by $B M O$ when $p=2$. For $(\alpha \cdot W)_{t}:=\int_{0}^{t} \alpha_{s} d W_{s}$ in $B M O$, the corresponding stochastic exponential is denoted by $\mathcal{E}_{t}(\alpha \cdot W)$.

We will now focus on the solvability of the following type of BSDEs with triangularly quadratic generators:

$$
\left\{\begin{aligned}
Y_{t}^{1}= & \xi^{1}+\int_{t}^{T}\left[\frac{1}{2}\left|Z_{s}^{1}\right|^{2}+Z_{s}^{1} l^{1}\left(s, Y_{s}\right)+h^{1}\left(s, Y_{s}, Z_{s}\right)\right] d s-\int_{t}^{T} Z_{s}^{1} d W_{s}, \\
Y_{t}^{i}= & \xi^{i}+\int_{t}^{T}\left[\frac{1}{2}\left|Z_{s}^{i}\right|^{2}+Z_{s}^{i} l^{i}\left(s, Y_{s}, Z_{s}\right)-k^{i}\left(s, Z_{s}\right)+h^{i}\left(s, Y_{s}, Z_{s}\right)\right] d s \\
& -\int_{t}^{T} Z_{s}^{i} d W_{s}, \quad i=2, \ldots, n,
\end{aligned}\right.
$$

where $\xi$ is $\mathbb{R}^{d}$-valued and $\mathcal{F}_{T}$-measurable random variable which is bounded. Let $C$ be a positive constant, we will make the following assumptions:

(A1) $l^{1}: \Omega \times[0, T] \times \mathbb{R}^{n} \rightarrow \mathbb{R}$ satisfies that $l^{1}(\cdot, y)$ is adapted for each $y \in \mathbb{R}^{n}$. Moreover, it holds that

$$
\begin{aligned}
& \left|l^{1}(t, y)\right| \leq C(1+|y|), \quad y \in \mathbb{R}^{n} ; \\
& \left|l^{1}(t, y)-l^{1}(t, \bar{y})\right| \leq C|y-\bar{y}|, \quad y, \bar{y} \in \mathbb{R}^{n}
\end{aligned}
$$

(A2) For $i=2, \ldots, n, l^{i}: \Omega \times[0, T] \times \mathbb{R}^{n} \times \mathbb{R}^{n \times d} \rightarrow \mathbb{R}$ satisfies that $l^{i}(\cdot, y, z)$ is adapted for each $y \in \mathbb{R}^{n}$ and $z \in \mathbb{R}^{n \times d}$. Moreover, it holds that

$$
\begin{aligned}
& \left|l^{i}(t, y, z)\right| \leq C(1+|y|), \quad y \in \mathbb{R}^{n}, z \in \mathbb{R}^{n \times d} ; \\
& \left|l^{i}(t, y, z)-l^{i}(t, \bar{y}, \bar{z})\right| \leq C|y-\bar{y}|+C \sum_{j=1}^{i-1}\left|z^{j}-\bar{z}^{j}\right|, \quad y, \bar{y} \in \mathbb{R}^{n}, z, \bar{z} \in \mathbb{R}^{n \times d} ;
\end{aligned}
$$


A type of globally solvable BSDEs with triangularly quadratic generators

(A3) For $i=2, \ldots, n, k^{i}: \Omega \times[0, T] \times \mathbb{R}^{n \times d} \rightarrow \mathbb{R}$ satisfies that $k^{i}(\cdot, z)$ is adapted for each $z \in \mathbb{R}^{n \times d}$. Moreover, it holds that

$$
\begin{aligned}
& 0 \leq k^{i}(t, z) \leq C\left(1+\sum_{j=1}^{i-1}\left|z^{j}\right|^{2}\right), \quad z \in \mathbb{R}^{n \times d} \\
& \left|k^{i}(t, z)-k^{i}(t, \bar{z})\right| \leq C \sum_{j=1}^{i-1}\left(1+\left|z^{j}\right|+\left|\bar{z}^{j}\right|\right)\left|z^{j}-\bar{z}^{j}\right|, \quad z, \bar{z} \in \mathbb{R}^{n \times d}
\end{aligned}
$$

(A4) $h: \Omega \times[0, T] \times \mathbb{R}^{n} \times \mathbb{R}^{n \times d} \rightarrow \mathbb{R}^{n}$ satisfies that $h(\cdot, y, z)$ is adapted for each $y \in \mathbb{R}^{n}$ and $z \in \mathbb{R}^{n \times d}$. Moreover, there exists $\alpha \in[-1,1)$ such that

$$
\begin{aligned}
& |h(t, y, z)| \leq C\left(1+|y|+|z|^{1+\alpha}\right), \quad y \in \mathbb{R}^{n}, z \in \mathbb{R}^{n \times d} ; \\
& |h(t, y, z)-h(t, \bar{y}, \bar{z})| \leq C|y-\bar{y}|+C\left(1+|z|^{\alpha^{+}}+|\bar{z}|^{\alpha^{+}}\right)|z-\bar{z}|,
\end{aligned}
$$

for $y, \bar{y} \in \mathbb{R}^{n}$ and $z, \bar{z} \in \mathbb{R}^{n \times d}$.

Remark 2.1. Assumptions (A2) and (A3) imply that $l^{i}(t, y, z)$ and $k^{i}(t, z)$ will only depend on the first $i-1$ components of $z$ for $i=2, \ldots, n$.

Our first main result ensures local existence and uniqueness for the triangularly quadratic BSDE (2.1). The proof is given in Section 3.

Theorem 2.2. Assume (A1)-(A4) hold, then there exist constants $T_{\eta}, C_{1}$ and $C_{2}$ only depending on $\alpha, C$ and $\|\xi\|_{\infty}$ such that for $T \leq T_{\eta}, B S D E$ (2.1) admits a unique solution $(Y, Z)$ such that $(Y, Z \cdot W) \in \mathcal{S}^{\infty}\left(\mathbb{R}^{n}\right) \times B M O$ with $\|Y\|_{\mathcal{S}^{\infty}} \leq C_{1}$ and $\|Z \cdot W\|_{B M O} \leq C_{2}$.

Next, we would like to find conditions under which Theorem 2.2 can be extended to obtain global solvability. In the present setting, under additional conditions, a pasting method based on the uniform bound of the value process allows to get global existence and uniqueness for the triangularly quadratic BSDE (2.1). The proof is given in Section 3.

Theorem 2.3. Assume (A1)-(A4) hold and additionally $h^{i} \leq 0$ and $\left|l^{i}\right| \leq C$ for $i=1, \ldots, n$, then BSDE (2.1) admits a unique solution $(Y, Z)$ such that $(Y, Z \cdot W) \in \mathcal{S}^{\infty}\left(\mathbb{R}^{n}\right) \times B M O$.

The sign of $k^{i}$ is critical to obtain our main results. Indeed, if the restriction on the sign of $k^{i}$ fails to hold true, we are able to construct a counterexample to the existence of solution of the triangularly quadratic BSDE based on the work of Frei and dos Reis [12].

Example 2.4. Assuming that $d=1$, then BSDE

$$
\left\{\begin{array}{l}
Y_{t}^{1}=\xi^{1}+\int_{t}^{T} \frac{1}{2}\left|Z_{s}^{1}\right|^{2} d s-\int_{t}^{T} Z_{s}^{1} d W_{s}, \\
Y_{t}^{2}=\int_{t}^{T}\left[\frac{1}{2}\left|Z_{s}^{2}\right|^{2}+\frac{\pi^{2}+1}{8}\left|Z_{s}^{1}\right|^{2}\right] d s-\int_{t}^{T} Z_{s}^{2} d W_{s},
\end{array}\right.
$$

has no solution for some $\xi^{1} \in L^{\infty}\left(\mathcal{F}_{T}\right)$. The details are given in Section 3.3.

Remark 2.5. Our results are closely related to Hu and Tang [14] and Xing and Žitković [28]. Compared with Xing and Žitković [28], we work under a non-Markovian setting. Compared with Hu and Tang [14], we allow two additional type of terms in our generator, i.e., $Z^{i} l^{i}(\cdot, Y, Z)$ for $i=1, \ldots, n$ and $k^{i}(\cdot, Z)$ for $i=2, \ldots, n$. The main difficulty is how to deal with the additional quadratic term $k^{i}(\cdot, Z)$. We overcome this difficulty by providing some critical estimates based on properties of BMO martingales. The detailed estimates are given in Section 3.

Remark 2.6. It is critical to obtain the uniform bound of the value process in order to obtain global existence. However, due to the additional quadratic term $k^{i}(\cdot, Z)$, the technique used in Hu and Tang [14] fails to work for our system of BSDEs. We provide 
A type of globally solvable BSDEs with triangularly quadratic generators

a new approach to obtain the uniform estimate for the value process by additionally assuming that $h^{i} \leq 0$ and $\left|l^{i}\right| \leq C$. If the additional quadratic term $k^{i}(\cdot, Z)$ vanishes, the global existence and uniqueness still hold true under the same assumption of $h^{i}$ as in $\mathrm{Hu}$ and Tang [14] by slightly modifying the technique in $\mathrm{Hu}$ and Tang [14].

\section{Triangularly quadratic BSDEs}

Before moving to the proofs of our main results, we would like to recall some classical results on $B M O$ spaces (see [18, Theorem 3.6] and [18, Corollary 2.1]).

Lemma 3.1. Let $a \cdot W \in B M O$ be such that $\|a \cdot W\|_{B M O} \leq \gamma$ for some $\gamma \geq 0$, and $\tilde{P}$ be given by $\frac{d \tilde{P}}{d P}:=\mathcal{E}_{T}(a \cdot W)$, under which $\tilde{W}=W-\int_{0}^{.} a_{s} d s$ is a Brownian motion. Then for every $b \cdot W \in B M O$, there exist two constants $\delta(\gamma)$ and $\Delta(\gamma)$ only depending on $\gamma$ such that

$$
\delta(\gamma)\|b \cdot W\|_{B M O}^{2} \leq\|b \cdot \tilde{W}\|_{B M O(\tilde{P})}^{2} \leq \Delta(\gamma)\|b \cdot W\|_{B M O}^{2}
$$

Lemma 3.2. For any $p \geq 1$, there is a generic constant $L_{p}>0$ such that for any uniformly integrable martingale $M$,

$$
\|M\|_{B M O_{p}}^{2} \leq L_{p}\|M\|_{B M O_{2}}^{2} .
$$

We consider the following function from $\mathbb{R}$ into itself defined by

$$
u(x)=e^{x}-1-x .
$$

It is easy to check that $u$ has the following properties

$$
u(x) \geq 0, \quad u(x) \geq|x|-1, \quad u^{\prime \prime}(x)-u^{\prime}(x)=1 .
$$

\subsection{Proof of Theorem 2.2}

Step 1. We first show that for $(y, z \cdot W) \in \mathcal{S}^{\infty}\left(\mathbb{R}^{n}\right) \times B M O$, the following BSDE

$$
Y_{s}^{1}=\xi^{1}+\int_{t}^{T}\left(\frac{1}{2}\left|Z_{s}^{1}\right|^{2}+Z_{s}^{1} l^{1}\left(s, y_{s}\right)+h^{1}\left(s, y_{s}, z_{s}\right)\right) d s-\int_{t}^{T} Z_{s}^{1} d W_{s}
$$

admits a unique solution $\left(Y^{1}, Z^{1}\right)$ such that $\left(Y^{1}, Z^{1} \cdot W\right) \in \mathcal{S}^{\infty}(\mathbb{R}) \times B M O$. Indeed, noting that $(y, z \cdot W) \in \mathcal{S}^{\infty}\left(\mathbb{R}^{n}\right) \times B M O$ and $\left|l^{1}(t, y)\right| \leq C(1+|y|)$, using Young's inequality, we obtain that for any $p \geq 1$,

$$
\begin{aligned}
& E^{Q^{1}}\left[e^{C p \int_{t}^{T}\left|z_{s}\right|^{1+\alpha} d s} \mid \mathcal{F}_{t}\right] \\
& \leq E^{Q^{1}}\left[e^{\int_{t}^{T} \frac{1+\alpha}{2} \frac{\left|z_{s}\right|^{2}}{(1+\alpha)\|z \cdot W\|_{B M O\left(Q^{1}\right)}^{2}}+\frac{1-\alpha}{2}(C p)^{\frac{1+\alpha}{1-\alpha}}\left((1+\alpha)\|z \cdot W\|_{B M O\left(Q^{1}\right)}^{2}\right)^{\frac{1+\alpha}{1-\alpha}} d s} \mid \mathcal{F}_{t}\right]
\end{aligned}
$$

where $Q^{1}$ is the equivalent probability measure given by $\frac{d Q^{1}}{d P}=\mathcal{E}_{T}\left(l^{1}(\cdot, y\right.$. $\left.) \cdot W\right)$. Applying John-Nirenberg inequality [18, Theorem 2.2], it holds that

$$
E^{Q^{1}}\left[e^{\int_{t}^{T} \frac{1+\alpha}{2} \frac{\left|z_{s}\right|^{2}}{(1+\alpha)\|z \cdot W\|_{B M O\left(Q^{1}\right)}^{2}}} d s \mid \mathcal{F}_{t}\right] \leq 2
$$

Therefore, combining with the fact that $\xi^{1}$ is bounded and $\left|h^{1}(t, y, z)\right| \leq C\left(1+|y|+|z|^{1+\alpha}\right)$, we have for any $p \geq 1$,

$$
E^{Q^{1}}\left[\left(e^{\xi^{1}+\int_{0}^{T} h^{1}\left(s, y_{s}, z_{s}\right) d s}\right)^{p}\right]<\infty
$$


A type of globally solvable BSDEs with triangularly quadratic generators

Hence, the following BSDE

$$
\hat{Y}_{s}^{1}=e^{\xi^{1}+\int_{0}^{T} h^{1}\left(s, y_{s}, z_{s}\right) d s}+\int_{t}^{T} \hat{Z}_{s}^{1} l^{1}\left(s, y_{s}\right) d s-\int_{t}^{T} \hat{Z}_{s}^{1} d W_{s}
$$

admits a unique solution $\left(\hat{Y}^{1}, \hat{Z}^{1}\right)$ with $\hat{Y}^{1}$ explicitly given by

$$
\hat{Y}_{t}^{1}=E^{Q^{1}}\left[e^{\xi^{1}+\int_{0}^{T} h^{1}\left(s, y_{s}, z_{s}\right) d s} \mid \mathcal{F}_{t}\right],
$$

and $\hat{Z}^{1}$ uniquely determined via martingale representation theorem through

$$
e^{\xi^{1}+\int_{0}^{T} h^{1}\left(s, y_{s}, z_{s}\right) d s}=E^{Q^{1}}\left[e^{\xi^{1}+\int_{0}^{T} h^{1}\left(s, y_{s}, z_{s}\right) d s}\right]+\int_{0}^{T} \hat{Z}_{t}^{1} d W_{t}^{Q^{1}} .
$$

Moreover $\hat{Y}^{1}>0$. Denoting $\tilde{Y}_{t}^{1}=\ln \hat{Y}_{t}^{1}$, we have

$$
\tilde{Y}_{t}^{1}=\left(\xi^{1}+\int_{0}^{T} h^{1}\left(s, y_{s}, z_{s}\right) d s\right)+\int_{t}^{T}\left[\frac{1}{2}\left|\frac{\hat{Z}_{s}^{1}}{\hat{Y}_{s}^{1}}\right|^{2}+\frac{\hat{Z}_{s}^{1}}{\hat{Y}_{s}^{1}} l^{1}\left(s, y_{s}\right)\right] d s-\int_{t}^{T} \frac{\hat{Z}_{s}^{1}}{\hat{Y}_{s}^{1}} d W_{s} .
$$

Let $Y_{t}^{1}=\tilde{Y}_{t}^{1}-\int_{0}^{t} h^{1}\left(s, y_{s}, z_{s}\right) d s$ and $Z_{t}^{1}=\frac{\hat{Z}_{t}^{1}}{\hat{Y}_{t}^{1}}$, then $\left(Y^{1}, Z^{1}\right)$ satisfies

$$
Y_{t}^{1}=\xi^{1}+\int_{t}^{T}\left(\frac{1}{2}\left|Z_{s}^{1}\right|^{2}+Z_{s}^{1} l^{1}\left(s, y_{s}\right)+h^{1}\left(s, y_{s}, z_{s}\right)\right) d s-\int_{t}^{T} Z_{s}^{1} d W_{s} .
$$

Now we will show that $\left(Y^{1}, Z^{1} \cdot W\right) \in \mathcal{S}^{\infty}(\mathbb{R}) \times B M O$. Actually, we have

$$
\begin{aligned}
Y_{t}^{1} & \leq \ln E^{Q^{1}}\left[e^{\left|\xi^{1}\right|+\int_{t}^{T}\left|h^{1}\left(s, y_{s}, z_{s}\right)\right| d s} \mid \mathcal{F}_{t}\right] \\
& \leq \ln E^{Q^{1}}\left[e^{\left|\xi^{1}\right|+\int_{t}^{T} C\left(1+\left|y_{s}\right|+\left|z_{s}\right|^{1+\alpha}\right) d s} \mid \mathcal{F}_{t}\right] \\
& \leq\left\|\xi^{1}\right\|_{\infty}+C\left(1+\|y\|_{\infty}\right)(T-t)+\ln E^{Q^{1}}\left[e^{C \int_{t}^{T}\left|z_{s}\right|^{1+\alpha} d s} \mid \mathcal{F}_{t}\right] .
\end{aligned}
$$

Therefore, it follows from (3.1) with $p=1$ and (3.2) that

$Y_{t}^{1} \leq\left\|\xi^{1}\right\|_{\infty}+C\left(1+\|y\|_{\infty}\right)(T-t)+\frac{1-\alpha}{2} C^{\frac{1+\alpha}{1-\alpha}}\left((1+\alpha)\|z \cdot W\|_{B M O\left(Q^{1}\right)}^{2}\right)^{\frac{1+\alpha}{1-\alpha}}(T-t)+\ln 2$.

On the other hand, it holds that

$$
\begin{aligned}
Y_{t}^{1} & =E^{Q^{1}}\left[\xi^{1}+\int_{t}^{T}\left(\frac{1}{2}\left|Z_{s}^{1}\right|^{2}+h^{1}\left(s, y_{s}, z_{s}\right)\right) d s \mid \mathcal{F}_{t}\right] \\
& \geq E^{Q^{1}}\left[\xi^{1}+\int_{t}^{T} h^{1}\left(s, y_{s}, z_{s}\right) d s \mid \mathcal{F}_{t}\right] \\
& \geq E^{Q^{1}}\left[-\left|\xi^{1}\right|-C \int_{t}^{T}\left(1+\left|y_{s}\right|+\left|z_{s}\right|^{1+\alpha}\right) d s \mid \mathcal{F}_{t}\right] \\
& \geq-\left\|\xi^{1}\right\|_{\infty}-C\left(1+\|y\|_{\infty}\right)(T-t)-C E^{Q^{1}}\left[\int_{t}^{T}\left|z_{s}\right|^{1+\alpha} d s \mid \mathcal{F}_{t}\right] .
\end{aligned}
$$

Using Young's inequality, we have

$$
\begin{aligned}
E^{Q^{1}}\left[\int_{t}^{T}\left|z_{s}\right|^{1+\alpha} d s \mid \mathcal{F}_{t}\right] \leq & \frac{1+\alpha}{2} \frac{1}{(1+\alpha)\|z \cdot W\|_{B M O\left(Q^{1}\right)}^{2}} E^{Q^{1}}\left[\int_{t}^{T}\left|z_{s}\right|^{2} d s \mid \mathcal{F}_{t}\right] \\
& +\frac{1-\alpha}{2}\left((1+\alpha)\|z \cdot W\|_{B M O\left(Q^{1}\right)}^{2}\right)^{\frac{1+\alpha}{1-\alpha}}(T-t) \\
\leq & \frac{1}{2}+\frac{1-\alpha}{2}\left((1+\alpha)\|z \cdot W\|_{B M O\left(Q^{1}\right)}^{2}\right)^{\frac{1+\alpha}{1-\alpha}}(T-t) .
\end{aligned}
$$


A type of globally solvable BSDEs with triangularly quadratic generators

Hence, it holds that

$$
\begin{aligned}
\left|Y_{t}^{1}\right| \leq & \left\|\xi^{1}\right\|_{\infty}+C\left(1+\|y\|_{\infty}\right)(T-t)+\ln 2+\frac{1}{2} \\
& +\frac{1-\alpha}{2}\left(C^{\frac{1+\alpha}{1-\alpha}}+1\right)\left((1+\alpha)\|z \cdot W\|_{B M O\left(Q^{1}\right)}^{2}\right)^{\frac{1+\alpha}{1-\alpha}}(T-t) .
\end{aligned}
$$

Applying Itô's formula to $u\left(Y_{t}^{1}\right)$, we obtain that

$$
\begin{aligned}
& u\left(Y_{t}^{1}\right)=u\left(\xi^{1}\right)-\int_{t}^{T} u^{\prime}\left(Y_{s}^{1}\right) Z_{s}^{1} d W_{s} \\
& +\int_{t}^{T}\left(u^{\prime}\left(Y_{s}^{1}\right)\left(\frac{1}{2}\left|Z_{s}^{1}\right|^{2}+Z_{s}^{1} l^{1}\left(s, y_{s}\right)+h^{1}\left(s, y_{s}, z_{s}\right)\right)-\frac{1}{2} u^{\prime \prime}\left(Y_{s}^{1}\right)\left|Z_{s}^{1}\right|^{2}\right) d s \\
& =u\left(\xi^{1}\right)-\int_{t}^{T} u^{\prime}\left(Y_{s}^{1}\right) Z_{s}^{1} d W_{s}-\frac{1}{2} \int_{t}^{T}\left|Z_{s}^{1}\right|^{2} d s \\
& +\int_{t}^{T} u^{\prime}\left(Y_{s}^{1}\right)\left(Z_{s}^{1} l^{1}\left(s, y_{s}\right)+h^{1}\left(s, y_{s}, z_{s}\right)\right) d s .
\end{aligned}
$$

Therefore, we have

$$
\begin{aligned}
& E^{Q^{1}}\left[\frac{1}{2} \int_{t}^{T}\left|Z_{s}^{1}\right|^{2} d s \mid \mathcal{F}_{t}\right] \\
& \leq E^{Q^{1}}\left[u\left(\xi^{1}\right)+\int_{t}^{T} u^{\prime}\left(Y_{s}^{1}\right) h^{1}\left(s, y_{s}, z_{s}\right) d s \mid \mathcal{F}_{t}\right] \\
& \leq e^{\left\|\xi^{1}\right\|_{\infty}}+\left\|\xi^{1}\right\|_{\infty}+C e^{\left\|\xi^{1}\right\|_{\infty}} E^{Q^{1}}\left[\int_{t}^{T}\left(1+\left|y_{s}\right|+\left|z_{s}\right|^{1+\alpha}\right) d s \mid \mathcal{F}_{t}\right] \\
& \leq e^{\left\|\xi^{1}\right\|_{\infty}}+\left\|\xi^{1}\right\|_{\infty}+C e^{\left\|\xi^{1}\right\|_{\infty}}\left(1+\|y\|_{\infty}\right)(T-t)+C e^{\left\|\xi^{1}\right\|_{\infty}} E^{Q^{1}}\left[\int_{t}^{T}\left|z_{s}\right|^{1+\alpha} d s \mid \mathcal{F}_{t}\right] .
\end{aligned}
$$

Noting the inequality (3.3), it holds that

$$
\begin{aligned}
E^{Q^{1}}\left[\frac{1}{2} \int_{t}^{T}\left|Z_{s}^{1}\right|^{2} d s \mid \mathcal{F}_{t}\right] \leq & \left(1+\frac{C}{2}\right) e^{\left\|\xi^{1}\right\|_{\infty}}+\left\|\xi^{1}\right\|_{\infty}+C e^{\left\|\xi^{1}\right\|_{\infty}}\left(1+\|y\|_{\infty}\right)(T-t) \\
& +\frac{1-\alpha}{2} C e^{\left\|\xi^{1}\right\|_{\infty}}\left((1+\alpha)\|z \cdot W\|_{B M O\left(Q^{1}\right)}^{2}\right)^{\frac{1+\alpha}{1-\alpha}}(T-t) .
\end{aligned}
$$

Therefore by denoting $\Delta:=\Delta\left(C\left(1+\|y\|_{\mathcal{S}^{\infty}}\right) \sqrt{T}\right)$ and $\delta:=\delta\left(C\left(1+\|y\|_{\mathcal{S}^{\infty}}\right) \sqrt{T}\right)$, we obtain that

$\left\|Y^{1}\right\|_{\mathcal{S}^{\infty}} \leq\left\|\xi^{1}\right\|_{\infty}+C\left(1+\|y\|_{\infty}\right) T+\ln 2+\frac{1}{2}+\frac{1-\alpha}{2}\left(C^{\frac{1+\alpha}{1-\alpha}}+1\right)\left((1+\alpha) \Delta\|z \cdot W\|_{B M O}^{2}\right)^{\frac{1+\alpha}{1-\alpha}} T$

and

$$
\begin{aligned}
\left\|Z^{1} \cdot W\right\|_{B M O}^{2} \leq & \frac{2}{\delta}\left(\left(1+\frac{C}{2}\right) e^{\left\|\xi^{1}\right\|_{\infty}}+\left\|\xi^{1}\right\|_{\infty}+C e^{\left\|\xi^{1}\right\|_{\infty}}\left(1+\|y\|_{\infty}\right) T\right. \\
& \left.+\frac{1-\alpha}{2} C e^{\left\|\xi^{1}\right\|_{\infty}}\left((1+\alpha) \Delta\|z \cdot W\|_{B M O}^{2}\right)^{\frac{1+\alpha}{1-\alpha}} T\right) .
\end{aligned}
$$


A type of globally solvable BSDEs with triangularly quadratic generators

Step 2. Similar to the first step, it is easy to check that

$$
Y_{t}^{2}=\xi^{2}+\int_{t}^{T}\left(\frac{1}{2}\left|Z_{s}^{2}\right|^{2}+Z_{s}^{2} l^{2}\left(s, y_{s}, Z_{s}^{1}\right)-k^{2}\left(s, Z_{s}^{1}\right)+h^{2}\left(s, y_{s}, z_{s}\right)\right) d t-\int_{t}^{T} Z_{s}^{2} d W_{s}
$$

admits a unique solution $\left(Y^{2}, Z^{2}\right)$ such that $\left(Y^{2}, Z^{2} \cdot W\right) \in \mathcal{S}^{\infty}(\mathbb{R}) \times B M O$. Moreover, we have

$$
\begin{aligned}
\left|Y_{t}^{2}\right| \leq & \left\|\xi^{2}\right\|_{\infty}+C\left(2+\|y\|_{\infty}\right)(T-t)+C\left\|Z^{1} \cdot W\right\|_{B M O\left(Q^{2}\right)}^{2}+\ln 2+\frac{1}{2} \\
& +\frac{1-\alpha}{2}\left(C^{\frac{1+\alpha}{1-\alpha}}+1\right)\left((1+\alpha)\|z \cdot W\|_{B M O\left(Q^{2}\right)}^{2}\right)^{\frac{1+\alpha}{1-\alpha}}(T-t)
\end{aligned}
$$

and

$$
\begin{aligned}
& E^{Q^{2}}\left[\frac{1}{2} \int_{t}^{T}\left|Z_{s}^{2}\right|^{2} d s \mid \mathcal{F}_{t}\right] \\
& \leq\left(1+\frac{C}{2}\right) e^{\left\|\xi^{2}\right\|_{\infty}}+\left\|\xi^{2}\right\|_{\infty}+C e^{\left\|\xi^{2}\right\|_{\infty}\left(2+\|y\|_{\infty}\right)(T-t)} \\
& \quad+\frac{1-\alpha}{2} C e^{\left\|\xi^{2}\right\|_{\infty}}\left((1+\alpha)\|z \cdot W\|_{B M O\left(Q^{2}\right)}^{2}\right)^{\frac{1+\alpha}{1-\alpha}}(T-t)+C\left\|Z^{1} \cdot W\right\|_{B M O\left(Q^{2}\right)}^{2}
\end{aligned}
$$

where $Q^{2}$ is the equivalent probability measure given by $\frac{d Q^{2}}{d P}=\mathcal{E}_{T}\left(l^{2}\left(\cdot, y ., Z^{1}\right) \cdot W\right)$. Therefore we obtain that

$$
\begin{aligned}
\left\|Y^{2}\right\|_{\mathcal{S}^{\infty}} \leq & \left\|\xi^{2}\right\|_{\infty}+C\left(2+\|y\|_{\infty}\right) T+C \Delta\left\|Z^{1} \cdot W\right\|_{B M O}^{2}+\ln 2+\frac{1}{2} \\
& +\frac{1-\alpha}{2}\left(C^{\frac{1+\alpha}{1-\alpha}}+1\right)\left((1+\alpha) \Delta\|z \cdot W\|_{B M O}^{2}\right)^{\frac{1+\alpha}{1-\alpha}} T
\end{aligned}
$$

and

$$
\begin{aligned}
\left\|Z^{2} \cdot W\right\|_{B M O}^{2} \leq & \frac{2}{\delta}\left(\left(1+\frac{C}{2}\right) e^{\left\|\xi^{2}\right\|_{\infty}}+\left\|\xi^{2}\right\|_{\infty}+C e^{\left\|\xi^{2}\right\|_{\infty}}\left(2+\|y\|_{\infty}\right) T\right. \\
& \left.+\frac{1-\alpha}{2} C e^{\left\|\xi^{2}\right\|_{\infty}}\left((1+\alpha) \Delta\|z \cdot W\|_{B M O}^{2}\right)^{\frac{1+\alpha}{1-\alpha}} T+C \Delta\left\|Z^{1} \cdot W\right\|_{B M O}^{2}\right) .
\end{aligned}
$$

Recursively, we have that for $i=3, \ldots, n$, the following BSDE

$$
Y_{t}^{i}=\xi^{i}+\int_{t}^{T}\left(\frac{1}{2}\left|Z_{s}^{i}\right|^{2}+Z_{s}^{i} l^{i}\left(s, y_{s}, Z_{s}\right)-k^{i}\left(s, Z_{s}\right)+h^{i}\left(s, y_{s}, z_{s}\right)\right) d t-\int_{t}^{T} Z_{s}^{i} d W_{s}
$$

admits a unique solution $\left(Y^{i}, Z^{i}\right)$ such that $\left(Y^{i}, Z^{i} \cdot W\right) \in \mathcal{S}^{\infty}(\mathbb{R}) \times B M O$. Moreover, we have

$$
\begin{aligned}
\left|Y_{t}^{i}\right| \leq & \left\|\xi^{i}\right\|_{\infty}+C\left(2+\|y\|_{\infty}\right)(T-t)+C \sum_{j=1}^{i-1}\left\|Z^{j} \cdot W\right\|_{B M O\left(Q^{i}\right)}^{2}+\ln 2+\frac{1}{2} \\
& +\frac{1-\alpha}{2}\left(C^{\frac{1+\alpha}{1-\alpha}}+1\right)\left((1+\alpha)\|z \cdot W\|_{B M O\left(Q^{i}\right)^{\frac{1+\alpha}{1-\alpha}}}^{2}(T-t)\right.
\end{aligned}
$$

and 
A type of globally solvable BSDEs with triangularly quadratic generators

$$
\begin{aligned}
& E^{Q^{i}}\left[\frac{1}{2} \int_{t}^{T}\left|Z_{s}^{i}\right|^{2} d s \mid \mathcal{F}_{t}\right] \\
& \leq\left(1+\frac{C}{2}\right) e^{\left\|\xi^{i}\right\|_{\infty}}+\left\|\xi^{i}\right\|_{\infty}+C e^{\left\|\xi^{i}\right\|_{\infty}}\left(2+\|y\|_{\infty}\right)(T-t) \\
& \quad+\frac{1-\alpha}{2} C e^{\left\|\xi^{i}\right\|_{\infty}}\left((1+\alpha)\|z \cdot W\|_{B M O\left(Q^{i}\right)}^{2}\right)^{\frac{1+\alpha}{1-\alpha}}(T-t)+C \sum_{j=1}^{i-1}\left\|Z^{j} \cdot W\right\|_{B M O\left(Q^{i}\right)}^{2}
\end{aligned}
$$

where $Q^{i}$ is the equivalent probability measure given by $\frac{d Q^{i}}{d P}=\mathcal{E}_{T}\left(l^{i}(\cdot, y, Z\right.$. $\left.) \cdot W\right)$. Therefore we obtain that

$$
\begin{aligned}
\left\|Y^{i}\right\|_{\mathcal{S} \infty} \leq & \left\|\xi^{i}\right\|_{\infty}+C\left(2+\|y\|_{\infty}\right) T+C \Delta \sum_{j=1}^{i-1}\left\|Z^{j} \cdot W\right\|_{B M O}^{2}+\ln 2+\frac{1}{2} \\
& +\frac{1-\alpha}{2}\left(C^{\frac{1+\alpha}{1-\alpha}}+1\right)\left((1+\alpha) \Delta\|z \cdot W\|_{B M O}^{2}\right)^{\frac{1+\alpha}{1-\alpha}} T
\end{aligned}
$$

and

$$
\begin{aligned}
\left\|Z^{i} \cdot W\right\|_{B M O}^{2} \leq & \frac{2}{\delta}\left(\left(1+\frac{C}{2}\right) e^{\left\|\xi^{i}\right\|_{\infty}}+\left\|\xi^{i}\right\|_{\infty}+C e^{\left\|\xi^{i}\right\|_{\infty}}\left(2+\|y\|_{\infty}\right) T\right. \\
& \left.+\frac{1-\alpha}{2} C e^{\left\|\xi^{i}\right\|_{\infty}}\left((1+\alpha) \Delta\|z \cdot W\|_{B M O}^{2}\right)^{\frac{1+\alpha}{1-\alpha}} T+C \Delta \sum_{j=1}^{i-1}\left\|Z^{j} \cdot W\right\|_{B M O}^{2}\right) .
\end{aligned}
$$

Step 3. We will denote

$$
\begin{aligned}
& \Delta^{*}=\Delta(C), \\
& \delta^{*}=\delta(C), \\
& A=n\left(\|\xi\|_{\infty}+2+C+\frac{C^{\frac{1+\alpha}{1-\alpha}}}{2}+\frac{2 n^{3} C \Delta^{*}}{\delta^{*}}\left(1+\left(\frac{2 C \Delta^{*}}{\delta^{*}}\right)^{n}\right)\left((1+2 C) e^{\|\xi\|_{\infty}}+\|\xi\|_{\infty}\right)\right), \\
& B=\sqrt{\frac{2 n^{3}}{\delta^{*}}\left(1+\left(\frac{2 C \Delta^{*}}{\delta^{*}}\right)^{n}\right)\left((1+2 C) e^{\left.\|\xi\|_{\infty}+\|\xi\|_{\infty}\right)},\right.} \\
& \eta=\frac{1}{(2+A)^{2}} \wedge \frac{1}{(1-\alpha)\left((1+\alpha) \Delta^{*} B^{2}\right)^{\frac{1+\alpha}{1-\alpha}}} .
\end{aligned}
$$

Assuming that $T \leq \eta$, for $(y, z \cdot W) \in \mathcal{S}^{\infty}\left(\mathbb{R}^{n}\right) \times B M O$ such that $\|y\|_{\mathcal{S}^{\infty}} \leq A$ and $\|z \cdot W\|_{B M O} \leq B$, it follows from Step 1 and Step 2 that the following BSDE

$$
\left\{\begin{aligned}
Y_{t}^{1}= & \xi^{1}+\int_{t}^{T}\left[\frac{1}{2}\left|Z_{s}^{1}\right|^{2}+Z_{s}^{1} l^{1}\left(s, y_{s}\right)+h^{1}\left(s, y_{s}, z_{s}\right)\right] d s-\int_{t}^{T} Z_{s}^{1} d W_{s} \\
Y_{t}^{i}= & \xi^{i}+\int_{t}^{T}\left[\frac{1}{2}\left|Z_{s}^{i}\right|^{2}+Z_{s}^{i} l^{i}\left(s, y_{s}, Z_{s}\right)-k^{i}\left(s, Z_{s}\right)+h^{i}\left(s, y_{s}, z_{s}\right)\right] d t \\
& -\int_{t}^{T} Z_{t}^{i} d W_{t}, \quad i=2, \ldots, n,
\end{aligned}\right.
$$

admits a unique solution $(Y, Z)$ such that $(Y, Z \cdot W) \in \mathcal{S}^{\infty}\left(\mathbb{R}^{n}\right) \times B M O$. Moreover from Lemma 3.1, $\Delta$ and $\delta$ can be replaced by $\Delta^{*}$ and $\delta^{*}$ respectively since it holds that

$$
C\left(1+\|y\|_{\mathcal{S}^{\infty}}\right) \sqrt{T} \leq C(1+A) \sqrt{\eta} \leq \frac{1+A}{2+A} C \leq C .
$$


A type of globally solvable BSDEs with triangularly quadratic generators

Therefore, it holds that

$$
\begin{aligned}
\left\|Y^{1}\right\|_{\mathcal{S}^{\infty}} & \\
& \leq\left\|\xi^{1}\right\|_{\infty}+C\left(1+\|y\|_{\infty}\right) T+\ln 2+\frac{1}{2}+\frac{1-\alpha}{2}\left(C^{\frac{1+\alpha}{1-\alpha}}+1\right)\left((1+\alpha) \Delta^{*}\|z \cdot W\|_{B M O}^{2}\right)^{\frac{1+\alpha}{1-\alpha}} T \\
& \leq\left\|\xi^{1}\right\|_{\infty}+C(1+A) T+\ln 2+\frac{1}{2}+\frac{1-\alpha}{2}\left(C^{\frac{1+\alpha}{1-\alpha}}+1\right)\left((1+\alpha) \Delta^{*} B^{2}\right)^{\frac{1+\alpha}{1-\alpha}} T \\
& \leq\left\|\xi^{1}\right\|_{\infty}+2+C+\frac{C^{\frac{1+\alpha}{1-\alpha}}}{2}
\end{aligned}
$$

and

$$
\begin{aligned}
\left\|Z^{1} \cdot W\right\|_{B M O}^{2} \leq & \frac{2}{\delta^{*}}\left(\left(1+\frac{C}{2}\right) e^{\left\|\xi^{1}\right\|_{\infty}}+\left\|\xi^{1}\right\|_{\infty}+C e^{\left\|\xi^{1}\right\|_{\infty}}\left(1+\|y\|_{\infty}\right) T\right. \\
& \left.+\frac{1-\alpha}{2} C e^{\left\|\xi^{1}\right\|_{\infty}}\left((1+\alpha) \Delta^{*}\|z \cdot W\|_{B M O}^{2}\right)^{\frac{1+\alpha}{1-\alpha}} T\right) \\
\leq & \frac{2}{\delta^{*}}\left(\left(1+\frac{C}{2}\right) e^{\left\|\xi^{1}\right\|_{\infty}}+\left\|\xi^{1}\right\|_{\infty}+C e^{\left\|\xi^{1}\right\|_{\infty}}(1+A) T\right. \\
& \left.+\frac{1-\alpha}{2} C e^{\left\|\xi^{1}\right\|_{\infty}}\left((1+\alpha) \Delta^{*} B^{2}\right)^{\frac{1+\alpha}{1-\alpha}} T\right) \\
\leq & \frac{2}{\delta^{*}}\left((1+2 C) e^{\left\|\xi^{1}\right\|_{\infty}}+\left\|\xi^{1}\right\|_{\infty}\right) .
\end{aligned}
$$

Similarly, noting that $\|y\|_{\mathcal{S}^{\infty}} \leq A$ and $\|z \cdot W\|_{B M O} \leq B$, we have

$$
\begin{aligned}
\left\|Y^{i}\right\|_{\mathcal{S} \infty} \leq & \left\|\xi^{i}\right\|_{\infty}+C\left(2+\|y\|_{\infty}\right) T+C \Delta^{*} \sum_{j=1}^{i-1}\left\|Z^{j} \cdot W\right\|_{B M O}^{2}+\ln 2+\frac{1}{2} \\
& +\frac{1-\alpha}{2}\left(C^{\frac{1+\alpha}{1-\alpha}}+1\right)\left((1+\alpha) \Delta^{*}\|z \cdot W\|_{B M O}^{2}\right)^{\frac{1+\alpha}{1-\alpha}} T \\
\leq & \left\|\xi^{i}\right\|_{\infty}+C(2+A) T+C \Delta^{*} \sum_{j=1}^{i-1}\left\|Z^{j} \cdot W\right\|_{B M O}^{2}+\ln 2+\frac{1}{2} \\
& +\frac{1-\alpha}{2}\left(C^{\frac{1+\alpha}{1-\alpha}}+1\right)\left((1+\alpha) \Delta^{*} B^{2}\right)^{\frac{1+\alpha}{1-\alpha}} T \\
\leq & \left\|\xi^{i}\right\|_{\infty}+2+C+\frac{C^{\frac{1+\alpha}{1-\alpha}}}{2}+C \Delta^{*} \sum_{j=1}^{i-1}\left\|Z^{j} \cdot W\right\|_{B M O}^{2}
\end{aligned}
$$

and

$$
\begin{aligned}
\left\|Z^{i} \cdot W\right\|_{B M O}^{2} \leq & \frac{2}{\delta^{*}}\left(\left(1+\frac{C}{2}\right) e^{\left\|\xi^{i}\right\|_{\infty}}+\left\|\xi^{i}\right\|_{\infty}+C e^{\left\|\xi^{i}\right\|_{\infty}}\left(2+\|y\|_{\infty}\right) T\right. \\
& \left.+\frac{1-\alpha}{2} C e^{\left\|\xi^{i}\right\|_{\infty}}\left((1+\alpha) \Delta_{i}\|z \cdot W\|_{B M O}^{2}\right)^{\frac{1+\alpha}{1-\alpha}} T+C \Delta^{*} \sum_{j=1}^{i-1}\left\|Z^{j} \cdot W\right\|_{B M O}^{2}\right) \\
\leq & \frac{2}{\delta^{*}}\left(\left(1+\frac{C}{2}\right) e^{\left\|\xi^{i}\right\|_{\infty}}+\left\|\xi^{i}\right\|_{\infty}+C e^{\left\|\xi^{i}\right\|_{\infty}}(2+A) T\right. \\
& \left.+\frac{1-\alpha}{2} C e^{\left\|\xi^{i}\right\|_{\infty}}\left((1+\alpha) \Delta_{i} B^{2}\right)^{\frac{1+\alpha}{1-\alpha}} T+C \Delta^{*} \sum_{j=1}^{i-1}\left\|Z^{j} \cdot W\right\|_{B M O}^{2}\right) \\
\leq & \frac{2}{\delta^{*}}\left((1+2 C) e^{\left\|\xi^{i}\right\|_{\infty}}+\left\|\xi^{i}\right\|_{\infty}+C \Delta^{*} \sum_{j=1}^{i-1}\left\|Z^{j} \cdot W\right\|_{B M O}^{2}\right)
\end{aligned}
$$


A type of globally solvable BSDEs with triangularly quadratic generators

Thus, we obtain recursively

$$
\begin{aligned}
\left\|Z^{i} \cdot W\right\|_{B M O}^{2} & \leq \frac{2 i}{\delta^{*}} \sum_{j=1}^{i}\left(\frac{2 C \Delta^{*}}{\delta^{*}}\right)^{i-j}\left((1+2 C) e^{\left\|\xi^{i}\right\|_{\infty}}+\left\|\xi^{i}\right\|_{\infty}\right) \\
& \leq \frac{2 i}{\delta^{*}} \sum_{j=1}^{i}\left(\frac{2 C \Delta^{*}}{\delta^{*}}\right)^{i-j}\left((1+2 C) e^{\|\xi\|_{\infty}}+\|\xi\|_{\infty}\right) \\
& \leq \frac{2 n^{2}}{\delta^{*}}\left(1+\left(\frac{2 C \Delta^{*}}{\delta^{*}}\right)^{n}\right)\left((1+2 C) e^{\|\xi\|_{\infty}}+\|\xi\|_{\infty}\right)
\end{aligned}
$$

and

$$
\begin{aligned}
\left\|Y^{i}\right\|_{\mathcal{S}^{\infty}} & \leq\left\|\xi^{i}\right\|_{\infty}+2+C+\frac{C^{\frac{1+\alpha}{1-\alpha}}}{2}+C \Delta^{*} \sum_{j=1}^{i-1}\left\|Z^{j} \cdot W\right\|_{B M O}^{2} \\
& \leq\|\xi\|_{\infty}+2+C+\frac{C^{\frac{1+\alpha}{1-\alpha}}}{2}+\frac{2 n^{3} C \Delta^{*}}{\delta^{*}}\left(1+\left(\frac{2 C \Delta^{*}}{\delta^{*}}\right)^{n}\right)\left((1+2 C) e^{\|\xi\|_{\infty}}+\|\xi\|_{\infty}\right)
\end{aligned}
$$

Thus, it holds that $\|Y\|_{\mathcal{S}^{\infty}} \leq A$ and $\|Z \cdot W\|_{B M O} \leq B$.

Step 4. We will denote

$$
\begin{aligned}
& \bar{\Delta}:=\Delta(\sqrt{2} C+2 \sqrt{2} B) \\
& \bar{\delta}:=\delta(\sqrt{2} C+2 \sqrt{2} B) \\
& \bar{A}=12 C^{2} T\left(1+\bar{\Delta} B^{2}\right) \\
& \bar{B}=18 C^{2} L_{4}^{2} \bar{\Delta}^{2} T^{1-\alpha^{+}}\left(3+2 \alpha^{+} L_{4} \bar{\Delta} B^{2}\right) \\
& \bar{C}=6 n C^{2} \bar{\Delta}^{2}\left(2 B^{2}+3 L_{4}^{2}\left(3+2 L_{4} \bar{\Delta} B^{2}\right)\right) \\
& \bar{\eta}_{1}=\frac{1}{24 C^{2}\left(1+\bar{\Delta} B^{2}\right)\left(n+\frac{\bar{C} n^{4}+n^{3}}{\bar{\delta}}\left(1+\left(\frac{\bar{C}}{\delta}\right)^{n}\right)\right)} \\
& \bar{\eta}_{2}=\left(\frac{1}{36 C^{2} L_{4}^{2} \bar{\Delta}^{2}\left(3+2 \alpha^{+} L_{4} \bar{\Delta} B^{2}\right)\left(n+\frac{\bar{C} n^{4}+n^{3}}{\bar{\delta}}\left(1+\left(\frac{\bar{C}}{\delta}\right)^{n}\right)\right)}\right)^{\frac{1}{1-\alpha^{+}}} .
\end{aligned}
$$

Assuming that $T \leq \eta \wedge \bar{\eta}_{1} \wedge \bar{\eta}_{2}$, for $(y, z \cdot W),(\bar{y}, \bar{z} \cdot W) \in \mathcal{S}^{\infty}\left(\mathbb{R}^{n}\right) \times B M O$ such that $\|y\|_{\mathcal{S}^{\infty}} \leq A,\|z \cdot W\|_{B M O} \leq B$ and $\|\bar{y}\|_{\mathcal{S}^{\infty}} \leq A,\|\bar{z} \cdot W\|_{B M O} \leq B$, it follows from Step 1, Step 2 and Step 3 that the following BSDE

$$
\left\{\begin{aligned}
Y_{t}^{1}= & \xi^{1}+\int_{t}^{T}\left(\frac{1}{2}\left|Z_{s}^{1}\right|^{2}+Z_{s}^{1} l^{1}\left(s, y_{s}\right)+h^{1}\left(s, y_{s}, z_{s}\right)\right) d s-\int_{t}^{T} Z_{s}^{1} d W_{s} \\
Y_{t}^{i}= & \xi^{i}+\int_{t}^{T}\left(\frac{1}{2}\left|Z_{s}^{i}\right|^{2}+Z_{s}^{i} l^{i}\left(s, y_{s}, Z_{s}\right)-k^{i}\left(s, Z_{s}\right)+h^{i}\left(s, y_{s}, z_{s}\right)\right) d s \\
& -\int_{t}^{T} Z_{s}^{i} d W_{s}, \quad i=2, \ldots, n
\end{aligned}\right.
$$

and

$$
\left\{\begin{array}{c}
\bar{Y}_{t}^{1}=\xi^{1}+\int_{t}^{T}\left(\frac{1}{2}\left|\bar{Z}_{s}^{1}\right|^{2}+\bar{Z}_{s}^{1} l^{1}\left(s, \bar{y}_{s}\right)+h^{1}\left(s, \bar{y}_{s}, \bar{z}_{s}\right)\right) d s-\int_{t}^{T} \bar{Z}_{s}^{1} d W_{s} \\
\bar{Y}_{t}^{i}=\xi^{i}+\int_{t}^{T}\left(\frac{1}{2}\left|\bar{Z}_{s}^{i}\right|^{2}+\bar{Z}_{s}^{i} l^{i}\left(s, \bar{y}_{s}, \bar{Z}_{s}\right)-k^{i}\left(s, \bar{Z}_{s}\right)+h^{i}\left(s, \bar{y}_{s}, \bar{z}_{s}\right)\right) d s \\
-\int_{t}^{T} Z_{s}^{i} d W_{s}, \quad i=2, \ldots, n
\end{array}\right.
$$


admit unique solutions $(Y, Z)$ and $(\bar{Y}, \bar{Z})$ respectively such that $(Y, Z \cdot W),(\bar{Y}, \bar{Z} \cdot W) \in$ $\mathcal{S}^{\infty}\left(\mathbb{R}^{n}\right) \times B M O$ with $\|Y\|_{\mathcal{S} \infty} \leq A,\|Z \cdot W\|_{B M O} \leq B$ and $\|\bar{Y}\|_{\mathcal{S}^{\infty}} \leq A,\|\bar{Z} \cdot W\|_{B M O} \leq B$. Then, we have

$$
\begin{aligned}
Y_{t}^{1}-\bar{Y}_{t}^{1}= & \int_{t}^{T}\left(\frac{1}{2}\left|Z_{s}^{1}\right|^{2}-\frac{1}{2}\left|\bar{Z}_{s}^{1}\right|^{2}+Z_{s}^{1} l^{1}\left(s, y_{s}\right)-\bar{Z}_{s}^{1} l^{1}\left(s, \bar{y}_{s}\right)+h^{1}\left(s, y_{s}, z_{s}\right)-h^{1}\left(s, \bar{y}_{s}, \bar{z}_{s}\right)\right) d s \\
& -\int_{t}^{T} Z_{s}^{1}-\bar{Z}_{s}^{1} d W_{s} \\
= & \int_{t}^{T} \bar{Z}_{s}^{1}\left(l^{1}\left(s, y_{s}\right)-l^{1}\left(s, \bar{y}_{s}\right)\right)+h^{1}\left(s, y_{s}, z_{s}\right)-h^{1}\left(s, \bar{y}_{s}, \bar{z}_{s}\right) d s-\int_{t}^{T} Z_{s}^{1}-\bar{Z}_{s}^{1} d \bar{W}_{s}^{1},
\end{aligned}
$$

where

$$
\bar{W}_{t}^{1}=W_{t}-\int_{0}^{t}\left(l^{1}\left(s, y_{s}\right)+\frac{1}{2}\left(Z_{s}^{1}+\bar{Z}_{s}^{1}\right)\right) d s
$$

is a Brownian motion under an equivalent probability measure $\bar{P}^{1}$ defined by

$$
\frac{d \bar{P}^{1}}{d P}=\mathcal{E}_{T}\left(\left(l^{1}(\cdot, y .)+\frac{1}{2}\left(Z_{.}^{1}+\bar{Z}_{.}^{1}\right)\right) \cdot W\right) .
$$

For any stopping time $\tau$ taking values in $[0, T]$, we have

$$
\begin{aligned}
& \left|Y_{\tau}^{1}-\bar{Y}_{\tau}^{1}\right|^{2}+\bar{E}^{1}\left[\int_{\tau}^{T}\left|Z_{s}^{1}-\bar{Z}_{s}^{1}\right|^{2} d s \mid \mathcal{F}_{\tau}\right] \\
& =\bar{E}^{1}\left[\left(\int_{\tau}^{T} \bar{Z}_{s}^{1}\left(l^{1}\left(s, y_{s}\right)-l^{1}\left(s, \bar{y}_{s}\right)\right)+h^{1}\left(s, y_{s}, z_{s}\right)-h^{1}\left(s, \bar{y}_{s}, \bar{z}_{s}\right) d s\right)^{2} \mid \mathcal{F}_{\tau}\right] \\
& \leq C^{2} \bar{E}^{1}\left[\left(\int_{\tau}^{T}\left(\left|\bar{Z}_{s}^{1}\right|\left|y_{s}-\bar{y}_{s}\right|+\left|y_{s}-\bar{y}_{s}\right|+\left(1+\left|z_{s}\right|^{\alpha^{+}}+\left|\bar{z}_{s}\right|^{\alpha^{+}}\right)\left|z_{s}-\bar{z}_{s}\right|\right) d s\right)^{2} \mid \mathcal{F}_{\tau}\right] \\
& \leq 3 C^{2}(T-\tau)^{2}\|y-\bar{y}\|_{\mathcal{S}^{\infty}}^{2}+3 C^{2}(T-\tau)\|y-\bar{y}\|_{\mathcal{S}^{\infty}}^{2} \bar{E}^{1}\left[\int_{\tau}^{T}\left|\bar{Z}_{s}^{1}\right|^{2} d s \mid \mathcal{F}_{\tau}\right] \\
& \quad+3 C^{2} \bar{E}^{1}\left[\int_{\tau}^{T}\left(1+\left|z_{s}\right|^{\alpha^{+}}+\left|\bar{z}_{s}\right|^{\alpha^{+}}\right)^{2} d s \int_{\tau}^{T}\left|z_{s}-\bar{z}_{s}\right|^{2} d s \mid \mathcal{F}_{\tau}\right] \\
& \leq 3 C^{2}(T-\tau)^{2}\|y-\bar{y}\|_{\mathcal{S}^{\infty}}^{2}+3 C^{2}(T-\tau)\|y-\bar{y}\|_{\mathcal{S} \infty}^{2} \bar{E}^{1}\left[\int_{\tau}^{T}\left|\bar{Z}_{s}^{1}\right|^{2} d s \mid \mathcal{F}_{\tau}\right] \\
& \quad+9 C^{2} \bar{E}^{1}\left[\left(\int_{\tau}^{T}\left(1+\left|z_{s}\right|^{2 \alpha^{+}}+\left|\bar{z}_{s}\right|^{2 \alpha^{+}}\right) d s\right)^{2} \mid \mathcal{F}_{\tau}\right]^{\frac{1}{2}}\left[\left(\int_{\tau}^{T}\left|z_{s}-\bar{z}_{s}\right|^{2} d s\right)^{2} \mid \mathcal{F}_{\tau}\right]^{\frac{1}{2}} .
\end{aligned}
$$

It follows from Lemma 3.1 and Lemma 3.2 that

$$
\bar{E}^{1}\left[\int_{\tau}^{T}\left|\bar{Z}_{s}^{1}\right|^{2} d s \mid \mathcal{F}_{\tau}\right] \leq\left\|\bar{Z}^{1} \cdot \bar{W}^{1}\right\|_{B M O\left(\bar{P}^{1}\right)}^{2} \leq \bar{\Delta}\left\|\bar{Z}^{1} \cdot W\right\|_{B M O}^{2}
$$

and

$$
\bar{E}^{1}\left[\left(\int_{\tau}^{T}\left|z_{s}-\bar{z}_{s}\right|^{2} d s\right)^{2} \mid \mathcal{F}_{\tau}\right]^{\frac{1}{2}} \leq L_{4}\left\|(z-\bar{z}) \cdot \bar{W}^{1}\right\|_{B M O\left(\bar{P}^{1}\right)}^{2} \leq L_{4} \bar{\Delta}\|(z-\bar{z}) \cdot W\|_{B M O}^{2}
$$


A type of globally solvable BSDEs with triangularly quadratic generators

and

$$
\begin{aligned}
& \bar{E}^{1}\left[\left(\int_{\tau}^{T}\left(1+\left|z_{s}\right|^{2 \alpha^{+}}+\left|\bar{z}_{s}\right|^{2 \alpha^{+}}\right) d s\right)^{2} \mid \mathcal{F}_{\tau}\right]^{\frac{1}{2}} \\
& \leq \bar{E}^{1}\left[\left(T+T^{1-\alpha^{+}}\left(\int_{\tau}^{T}\left|z_{s}\right|^{2} d s\right)^{\alpha^{+}}+T^{1-\alpha^{+}}\left(\int_{\tau}^{T}\left|\bar{z}_{s}\right|^{2} d s\right)^{\alpha^{+}}\right)^{2} \mid \mathcal{F}_{\tau}\right]^{\frac{1}{2}} \\
& \leq T^{1-\alpha^{+}} \bar{E}^{1}\left[\left(T^{\alpha^{+}}+\left(\int_{\tau}^{T}\left|z_{s}\right|^{2} d s\right)^{\alpha^{+}}+\left(\int_{\tau}^{T}\left|\bar{z}_{s}\right|^{2} d s\right)^{\alpha^{+}}\right)^{2} \mid \mathcal{F}_{\tau}\right]^{\frac{1}{2}} \\
& \leq T^{1-\alpha^{+}} \bar{E}^{1}\left[\left(T^{\alpha^{+}}+2-2 \alpha^{+}+\alpha^{+} \int_{\tau}^{T}\left|z_{s}\right|^{2} d s+\alpha^{+} \int_{\tau}^{T}\left|\bar{z}_{s}\right|^{2} d s\right)^{2} \mid \mathcal{F}_{\tau}\right]^{\frac{1}{2}} \\
& \leq T^{1-\alpha^{+}}\left(T^{\alpha^{+}}+2+\alpha^{+} \bar{E}^{1}\left[\left(\int_{\tau}^{T}\left|z_{s}\right|^{2} d s\right)^{2} \mid \mathcal{F}_{\tau}\right]^{\frac{1}{2}}+\alpha^{+} \bar{E}^{1}\left[\left(\int_{\tau}^{T}\left|\bar{z}_{s}\right|^{2} d s\right)^{2} \mid \mathcal{F}_{\tau}\right]\right. \\
& \leq T^{1-\alpha^{+}}\left(T^{\alpha^{+}}+2+\alpha^{+} L_{4}\|z \cdot W\|_{B M O\left(\bar{P}^{1}\right)}^{2}+\alpha^{+} L_{4}\|\bar{z} \cdot W\|_{B M O\left(\bar{P}^{1}\right)}^{2}\right) \\
& \leq T^{1-\alpha^{+}}\left(T^{\alpha^{+}}+2+\alpha^{+} L_{4} \bar{\Delta}\|z \cdot W\|_{B M O}^{2}+\alpha^{+} L_{4} \bar{\Delta}\|\bar{z} \cdot W\|_{B M O}^{2}\right) .
\end{aligned}
$$

Therefore, we have

$$
\begin{aligned}
& \left|Y_{\tau}^{1}-\bar{Y}_{\tau}^{1}\right|^{2}+\bar{E}^{1}\left[\int_{\tau}^{T}\left|Z_{s}^{1}-\bar{Z}_{s}^{1}\right|^{2} \mid \mathcal{F}_{\tau}\right] \\
& \leq 3 C^{2} T\left(T+\bar{\Delta} B^{2}\right)\|y-\bar{y}\|_{\mathcal{S}^{\infty}}^{2} \\
& \quad+9 C^{2} L_{4}^{2} \bar{\Delta}^{2} T^{1-\alpha^{+}}\left(T^{\alpha^{+}}+2+2 \alpha^{+} L_{4} \bar{\Delta} B^{2}\right)\|(z-\bar{z}) \cdot W\|_{B M O}^{2} .
\end{aligned}
$$

Hence

$$
\begin{aligned}
& \left\|Y^{1}-\bar{Y}^{1}\right\|_{\mathcal{S}^{\infty}}^{2}+\bar{\delta}\left\|Z_{s}^{1}-\bar{Z}_{s}^{1}\right\|_{B M O}^{2} \\
& \leq 6 C^{2} T\left(T+\bar{\Delta} B^{2}\right)\|y-\bar{y}\|_{\mathcal{S}^{\infty}}^{2} \\
& \quad+18 C^{2} L_{4}^{2} \bar{\Delta}^{2} T^{1-\alpha^{+}}\left(T^{\alpha^{+}}+2+2 \alpha^{+} L_{4} \bar{\Delta} B^{2}\right)\|(z-\bar{z}) \cdot W\|_{B M O}^{2} \\
& \leq \bar{A}\|y-\bar{y}\|_{\mathcal{S}^{\infty}}^{2}+\bar{B}\|(z-\bar{z}) \cdot W\|_{B M O}^{2} .
\end{aligned}
$$

Similarly, we could get that

$$
\begin{aligned}
& \left\|Y^{i}-\bar{Y}^{i}\right\|_{\mathcal{S}^{\infty}}^{2}+\bar{\delta}\left\|Z_{s}^{i}-\bar{Z}_{s}^{i}\right\|_{B M O}^{2} \\
& \leq 12 C^{2} T\left(T+\bar{\Delta} B^{2}\right)\|y-\bar{y}\|_{\mathcal{S}^{\infty}}^{2}+12 i C^{2} \bar{\Delta}^{2} B^{2} \sum_{j=1}^{i-1}\left\|\left(Z^{j}-\bar{Z}^{j}\right) \cdot W\right\|_{B M O}^{2} \\
& \quad+18 i C^{2} L_{4}^{2} \bar{\Delta}^{2}\left(T+2+2 L_{4} \bar{\Delta} B^{2}\right) \sum_{j=1}^{i-1}\left\|\left(Z^{j}-\bar{Z}^{j}\right) \cdot W\right\|_{B M O}^{2} \\
& \quad+18 C^{2} L_{4}^{2} \bar{\Delta}^{2} T^{1-\alpha^{+}}\left(T^{\alpha^{+}}+2+2 \alpha^{+} L_{4} \bar{\Delta} B^{2}\right)\|(z-\bar{z}) \cdot W\|_{B M O}^{2} \\
& \leq \bar{A}\|y-\bar{y}\|_{\mathcal{S}^{\infty}}^{2}+\bar{C} \sum_{j=1}^{i-1}\left\|\left(Z^{j}-\bar{Z}^{j}\right) \cdot W\right\|_{B M O}^{2}+\bar{B}\|(z-\bar{z}) \cdot W\|_{B M O}^{2} .
\end{aligned}
$$


A type of globally solvable BSDEs with triangularly quadratic generators

Thus, we obtain recursively

$$
\begin{aligned}
\left\|\left(Z_{s}^{i}-\bar{Z}_{s}^{i}\right) \cdot W\right\|_{B M O}^{2} & \leq \frac{i}{\bar{\delta}} \sum_{j=1}^{i}\left(\frac{\bar{C}}{\bar{\delta}}\right)^{i-j}\left(\bar{A}\|y-\bar{y}\|_{\mathcal{S}^{\infty}}^{2}+\bar{B}\|(z-\bar{z}) \cdot W\|_{B M O}^{2}\right) \\
& \leq \frac{n^{2}}{\bar{\delta}}\left(1+\left(\frac{\bar{C}}{\bar{\delta}}\right)^{n}\right)\left(\bar{A}\|y-\bar{y}\|_{\mathcal{S}^{\infty}}^{2}+\bar{B}\|(z-\bar{z}) \cdot W\|_{B M O}^{2}\right)
\end{aligned}
$$

and

$$
\left\|Y^{i}-\bar{Y}^{i}\right\|_{\mathcal{S}^{\infty}}^{2} \leq\left(1+\frac{\bar{C} n^{3}}{\bar{\delta}}\left(1+\left(\frac{\bar{C}}{\bar{\delta}}\right)^{n}\right)\right)\left(\bar{A}\|y-\bar{y}\|_{\mathcal{S}^{\infty}}^{2}+\bar{B}\|(z-\bar{z}) \cdot W\|_{B M O}^{2}\right)
$$

Therefore, we have

$$
\begin{aligned}
& \|Y-\bar{Y}\|_{\mathcal{S}^{\infty}}^{2}+\left\|\left(Z_{s}-\bar{Z}_{s}\right) \cdot W\right\|_{B M O}^{2} \\
& \leq\left(n+\frac{\bar{C} n^{4}+n^{3}}{\bar{\delta}}\left(1+\left(\frac{\bar{C}}{\bar{\delta}}\right)^{n}\right)\right)\left(\bar{A}\|y-\bar{y}\|_{\mathcal{S}^{\infty}}^{2}+\bar{B}\|(z-\bar{z}) \cdot W\|_{B M O}^{2}\right) \\
& \leq \frac{1}{2}\left(\|y-\bar{y}\|_{\mathcal{S}^{\infty}}^{2}+\|(z-\bar{z}) \cdot W\|_{B M O}^{2}\right),
\end{aligned}
$$

which implies BSDE (2.1) admits a unique solution $(Y, Z)$ such that $(Y, Z \cdot W) \in \mathcal{S}^{\infty}\left(\mathbb{R}^{n}\right) \times$ $B M O$ with $\|Y\|_{\mathcal{S}^{\infty}} \leq A$ and $\|Z \cdot W\|_{B M O} \leq B$.

\subsection{Proof of Theorem 2.3}

Let $\beta$. be the unique solution of the following ordinary differential equation

$$
\begin{aligned}
\beta_{t}= & n+n\left(1+4 n^{2}\left(1+\left(4 C e^{\|\xi\|_{\infty}}\right)^{n}\right)\right)\left(e^{\|\xi\|_{\infty}}+\|\xi\|_{\infty}+\left(C^{2} e^{2\|\xi\|_{\infty}}+2 C e^{\|\xi\|_{\infty}}\right) T\right) \\
& +\frac{1-\alpha}{8(1-\alpha)}\left(4(1+\alpha) n C e^{\|\xi\|_{\infty}}\left(1+4 n^{2}\left(1+\left(4 C e^{\|\xi\|_{\infty}}\right)^{n}\right)\right)\right)^{\frac{2}{1-\alpha}} T \\
& +n C e^{\|\xi\|_{\infty}}\left(1+4 n^{2}\left(1+\left(4 C e^{\|\xi\|_{\infty}}\right)^{n}\right)\right) \int_{t}^{T} \beta_{s} d s .
\end{aligned}
$$

It is easy to see that $\beta$. is a continuous and decreasing function. Define

$$
\lambda:=\sup _{t \in[0, T]} \beta_{t}=\beta_{0} .
$$

As $\|\xi\|_{\infty} \leq \lambda$, from Theorem 2.2, there exists $\eta_{\lambda}>0$ which only depends on $\lambda$ such that BSDE has a local solution $(Y, Z)$ on $\left[T-\eta_{\lambda}, T\right]$.

$$
\begin{aligned}
Y_{t}^{1} & =\xi^{1}+\int_{t}^{T} \frac{1}{2}\left|Z_{s}^{1}\right|^{2}+Z_{s}^{1} l^{1}\left(s, Y_{s}\right)+h^{1}\left(s, Y_{s}, Z_{s}\right) d s-\int_{t}^{T} Z_{s}^{1} d W_{s} \\
& \leq \xi^{1}+\int_{t}^{T} \frac{1}{2}\left|Z_{s}^{1}\right|^{2}+Z_{s}^{1} l^{1}\left(s, Y_{s}\right) d s-\int_{t}^{T} Z_{s}^{1} d W_{s}
\end{aligned}
$$

and

$$
\begin{aligned}
Y_{t}^{i} & =\xi^{i}+\int_{t}^{T} \frac{1}{2}\left|Z_{s}^{i}\right|^{2}+Z_{s}^{i} l^{i}\left(s, Y_{s}, Z_{s}\right)-k^{i}\left(s, Z_{s}\right)+h^{i}\left(s, Y_{s}, Z_{s}\right) d s-\int_{t}^{T} Z_{s}^{i} d W_{s} \\
& \leq \xi^{i}+\int_{t}^{T} \frac{1}{2}\left|Z_{s}^{i}\right|^{2}+Z_{s}^{i} l^{i}\left(s, Y_{s}, Z_{s}\right)-\int_{t}^{T} Z_{s}^{i} d W_{s}
\end{aligned}
$$


A type of globally solvable BSDEs with triangularly quadratic generators

Therefore, we have $Y_{t}^{i} \leq\left\|\xi^{i}\right\|_{\infty}$. Applying Itô's formula to $u\left(Y^{1}\right)$, we obtain that

$$
\begin{aligned}
u\left(Y_{t}^{1}\right) & u\left(\xi^{1}\right)-\int_{t}^{T} u^{\prime}\left(Y_{s}^{1}\right) Z_{s}^{1} d W_{s} \\
& +\int_{t}^{T}\left(u^{\prime}\left(Y_{s}^{1}\right)\left(\frac{1}{2}\left|Z_{s}^{1}\right|^{2}+Z_{s}^{1} l^{1}\left(Y_{s}\right)+h^{1}\left(s, Y_{s}, Z_{s}\right)\right)-\frac{1}{2} u^{\prime \prime}\left(Y_{s}^{1}\right)\left|Z_{s}^{1}\right|^{2}\right) d s \\
= & u\left(\xi^{1}\right)-\int_{t}^{T} u^{\prime}\left(Y_{s}^{1}\right) Z_{s}^{1} d W_{s}+\int_{t}^{T}\left(u^{\prime}\left(Y_{s}^{1}\right)\left(Z_{s}^{1} l^{1}\left(Y_{s}\right)+h^{1}\left(s, Y_{s}, Z_{s}\right)\right)-\frac{1}{2}\left|Z_{s}^{1}\right|^{2}\right) d s \\
\leq & e^{\left\|\xi^{1}\right\|_{\infty}}+\left\|\xi^{1}\right\|_{\infty}-\int_{t}^{T} u^{\prime}\left(Y_{s}^{1}\right) Z_{s}^{1} d W_{s} \\
& +\int_{t}^{T}\left(e^{\left\|\xi^{1}\right\|_{\infty}}\left(C\left|Z_{s}^{1}\right|+C\left(1+\left|Y_{s}\right|+\left|Z_{s}\right|^{1+\alpha}\right)\right)-\frac{1}{2}\left|Z_{s}^{1}\right|^{2}\right) d s \\
\leq & e^{\left\|\xi^{1}\right\|_{\infty}}+\left\|\xi^{1}\right\|_{\infty}-\int_{t}^{T} u^{\prime}\left(Y_{s}^{1}\right) Z_{s}^{1} d W_{s} \\
& +\int_{t}^{T}\left(\left(C^{2} e^{2\left\|\xi^{1}\right\|_{\infty}}+C e^{\left\|\xi^{1}\right\|_{\infty}}\left(1+\left|Y_{s}\right|+\left|Z_{s}\right|^{1+\alpha}\right)\right)-\frac{1}{4}\left|Z_{s}^{1}\right|^{2}\right) d s \\
\leq & e^{\|\xi\|_{\infty}}+\|\xi\|_{\infty}-\int_{t}^{T} u^{\prime}\left(Y_{s}^{1}\right) Z_{s}^{1} d W_{s} \\
& +\int_{t}^{T}\left(\left(C^{2} e^{2\|\xi\|_{\infty}}+C e^{\|\xi\|_{\infty}}\left(1+\left|Y_{s}\right|+\left|Z_{s}\right|^{1+\alpha}\right)\right)-\frac{1}{4}\left|Z_{s}^{1}\right|^{2}\right) d s
\end{aligned}
$$

Applying Itô's formula to $u\left(Y^{i}\right)$, we obtain that

$$
\begin{aligned}
& u\left(Y_{t}^{i}\right) \\
& =u\left(\xi^{i}\right)-\int_{t}^{T} u^{\prime}\left(Y_{s}^{i}\right) Z_{s}^{i} d W_{s} \\
& +\int_{t}^{T}\left(u^{\prime}\left(Y_{s}^{i}\right)\left(\frac{1}{2}\left|Z_{s}^{i}\right|^{2}+Z_{s}^{i} l^{i}\left(Y_{s}, Z_{s}\right)-k^{i}\left(s, Z_{s}\right)+h^{i}\left(s, Y_{s}, Z_{s}\right)\right)-\frac{1}{2} u^{\prime \prime}\left(Y_{s}^{i}\right)\left|Z_{s}^{i}\right|^{2}\right) d s \\
& =u\left(\xi^{i}\right)-\int_{t}^{T} u^{\prime}\left(Y_{s}^{i}\right) Z_{s}^{i} d W_{s} \\
& +\int_{t}^{T}\left(u^{\prime}\left(Y_{s}^{i}\right)\left(Z_{s}^{i} l^{i}\left(Y_{s}, Z_{s}\right)-k^{i}\left(s, Z_{s}\right)+h^{i}\left(s, Y_{s}, Z_{s}\right)\right)-\frac{1}{2}\left|Z_{s}^{i}\right|^{2}\right) d s \\
& \leq e^{\left\|\xi^{i}\right\|_{\infty}}+\left\|\xi^{i}\right\|_{\infty}-\int_{t}^{T} u^{\prime}\left(Y_{s}^{i}\right) Z_{s}^{i} d W_{s} \\
& +\int_{t}^{T}\left(e^{\left\|\xi^{i}\right\|_{\infty}}\left(C\left|Z_{s}^{i}\right|+C\left(1+\sum_{j=1}^{i-1}\left|Z_{s}^{j}\right|^{2}\right)+C\left(1+\left|Y_{s}\right|+\left|Z_{s}\right|^{1+\alpha}\right)\right)-\frac{1}{2}\left|Z_{s}^{i}\right|^{2}\right) d s \\
& \leq e^{\left\|\xi^{i}\right\|_{\infty}}+\left\|\xi^{i}\right\|_{\infty}-\int_{t}^{T} u^{\prime}\left(Y_{s}^{i}\right) Z_{s}^{i} d W_{s} \\
& +\int_{t}^{T}\left(C^{2} e^{2\left\|\xi^{i}\right\|_{\infty}}+C e^{\left\|\xi^{i}\right\|_{\infty}} \sum_{j=1}^{i-1}\left|Z_{s}^{j}\right|^{2}+C e^{\left\|\xi^{i}\right\|_{\infty}}\left(2+\left|Y_{s}\right|+\left|Z_{s}\right|^{1+\alpha}\right)-\frac{1}{4}\left|Z_{s}^{i}\right|^{2}\right) d s \\
& \leq e^{\|\xi\|_{\infty}}+\|\xi\|_{\infty}-\int_{t}^{T} u^{\prime}\left(Y_{s}^{i}\right) Z_{s}^{i} d W_{s} \\
& +\int_{t}^{T}\left(C^{2} e^{2\|\xi\|_{\infty}}+C e^{\|\xi\|_{\infty}} \sum_{j=1}^{i-1}\left|Z_{s}^{j}\right|^{2}+C e^{\|\xi\|_{\infty}}\left(2+\left|Y_{s}\right|+\left|Z_{s}\right|^{1+\alpha}\right)-\frac{1}{4}\left|Z_{s}^{i}\right|^{2}\right) d s
\end{aligned}
$$


A type of globally solvable BSDEs with triangularly quadratic generators

Recursively, taking conditional expectation with respect to $\mathcal{F}_{r}$ for $r \in\left[T-\eta_{\lambda}, t\right]$, we can show that

$$
\begin{aligned}
& E\left[u\left(Y_{t}^{i}\right) \mid \mathcal{F}_{r}\right] \\
& \leq\left(1+4 n^{2}\left(1+\left(4 C e^{\|\xi\|_{\infty}}\right)^{n}\right)\right)\left(e^{\|\xi\|_{\infty}}+\|\xi\|_{\infty}\right) \\
& \quad+E\left[\int_{t}^{T}\left(1+4 n^{2}\left(1+\left(4 C e^{\|\xi\|_{\infty}}\right)^{n}\right)\right)\left(C^{2} e^{2\|\xi\|_{\infty}}+2 C e^{\|\xi\|_{\infty}}\right) d s \mid \mathcal{F}_{r}\right] \\
& \quad+E\left[\int_{t}^{T}\left(1+4 n^{2}\left(1+\left(4 C e^{\|\xi\|_{\infty}}\right)^{n}\right)\right)\left(C e^{\|\xi\|_{\infty}}\left(\left|Y_{s}\right|+\left|Z_{s}\right|^{1+\alpha}\right)\right)-\frac{1}{4}\left|Z_{s}^{i}\right|^{2} d s \mid \mathcal{F}_{r}\right]
\end{aligned}
$$

Hence, it holds that

$$
\begin{aligned}
& E\left[\sum_{i=1}^{n} u\left(Y_{t}^{i}\right) \mid \mathcal{F}_{r}\right] \\
& \leq n\left(1+4 n^{2}\left(1+\left(4 C e^{\|\xi\|_{\infty}}\right)^{n}\right)\right)\left(e^{\|\xi\|_{\infty}}+\|\xi\|_{\infty}\right) \\
& +n E\left[\int_{t}^{T}\left(1+4 n^{2}\left(1+\left(4 C e^{\|\xi\|_{\infty}}\right)^{n}\right)\right)\left(C^{2} e^{2\|\xi\|_{\infty}}+2 C e^{\|\xi\|_{\infty}}\right) d s \mid \mathcal{F}_{r}\right] \\
& +E\left[\int_{t}^{T} n\left(1+4 n^{2}\left(1+\left(4 C e^{\|\xi\|_{\infty}}\right)^{n}\right)\right)\left(C e^{\|\xi\|_{\infty}}\left(\left|Y_{s}\right|+\left|Z_{s}\right|^{1+\alpha}\right)\right)-\frac{1}{4}\left|Z_{s}\right|^{2} d s \mid \mathcal{F}_{r}\right] \\
& \leq n\left(1+4 n^{2}\left(1+\left(4 C e^{\|\xi\|_{\infty}}\right)^{n}\right)\right)\left(e^{\|\xi\|_{\infty}}+\|\xi\|_{\infty}\right) \\
& +n E\left[\int_{t}^{T}\left(1+4 n^{2}\left(1+\left(4 C e^{\|\xi\|_{\infty}}\right)^{n}\right)\right)\left(C^{2} e^{2\|\xi\|_{\infty}}+2 C e^{\|\xi\|_{\infty}}\right) d s \mid \mathcal{F}_{r}\right] \\
& +E\left[\int_{t}^{T} n C e^{\|\xi\|_{\infty}}\left(1+4 n^{2}\left(1+\left(4 C e^{\|\xi\|_{\infty}}\right)^{n}\right)\right)\left|Y_{s}\right| d s \mid \mathcal{F}_{r}\right] \\
& +E\left[\int_{t}^{T} \frac{1-\alpha}{8(1-\alpha)}\left(4(1+\alpha) n C e^{\|\xi\|_{\infty}}\left(1+4 n^{2}\left(1+\left(4 C e^{\|\xi\|_{\infty}}\right)^{n}\right)\right)\right)^{\frac{2}{1-\alpha}} d s \mid \mathcal{F}_{r}\right] \\
& \quad-E\left[\int_{t}^{T} \frac{1}{8}\left|Z_{s}\right|^{2} d s \mid \mathcal{F}_{r}\right]
\end{aligned}
$$

Noting that

$$
u\left(Y_{t}^{i}\right) \geq\left|Y_{t}^{i}\right|-1,
$$

we have

$$
\begin{aligned}
E & {\left[\left|Y_{t}\right| \mid \mathcal{F}_{r}\right]+\frac{1}{8} E\left[\int_{t}^{T}\left|Z_{s}\right|^{2} \mid \mathcal{F}_{r}\right] } \\
\leq & n+n\left(1+4 n^{2}\left(1+\left(4 C e^{\|\xi\|_{\infty}}\right)^{n}\right)\right)\left(e^{\|\xi\|_{\infty}}+\|\xi\|_{\infty}+\left(C^{2} e^{2\|\xi\|_{\infty}}+2 C e^{\|\xi\|_{\infty}}\right) T\right) \\
& +\frac{1-\alpha}{8(1-\alpha)}\left(4(1+\alpha) n C e^{\|\xi\|_{\infty}}\left(1+4 n^{2}\left(1+\left(4 C e^{\|\xi\|_{\infty}}\right)^{n}\right)\right)\right)^{\frac{2}{1-\alpha}} T \\
& +n C e^{\|\xi\|_{\infty}}\left(1+4 n^{2}\left(1+\left(4 C e^{\|\xi\|_{\infty}}\right)^{n}\right)\right) \int_{t}^{T} E\left[\mid Y_{s} \| \mathcal{F}_{r}\right] d s .
\end{aligned}
$$

Hence, we deduce that

$$
E\left[\left|Y_{t}\right| \mid \mathcal{F}_{r}\right] \leq \beta_{t} .
$$


A type of globally solvable BSDEs with triangularly quadratic generators

Setting $r=t$, we have

$$
\left|Y_{t}\right| \leq \beta_{t} \leq \lambda
$$

Taking $T-\eta_{\lambda}$ as the terminal time and $Y_{T-\eta_{\lambda}}$ as terminal value, from Theorem 2.2, BSDE (2.1) has a local solution $(Y, Z)$ on $\left[T-2 \eta_{\lambda}, T-\eta_{\lambda}\right]$. Once again, we can deduce that $\left|Y_{t}\right| \leq \beta_{t}$, for $t \in\left[T-2 \eta_{\lambda}, T-\eta_{\lambda}\right]$. Repeating the preceding process, we can extend the pair $(Y, Z)$ to the whole interval $[0, T]$ within a finite steps such that $Y$ is uniformly bounded by $\lambda$. Moreover, similar to the above, we have

$$
\frac{1}{8} E\left[\int_{t}^{T}\left|Z_{s}\right|^{2} \mid \mathcal{F}_{t}\right] \leq \lambda
$$

Consequently, we have

$$
\|Z \cdot W\|_{B M O}^{2} \leq 8 \lambda
$$

Finally, the uniqueness on the given interval $[0, T]$ follows from Theorem 2.2 and a pasting technique.

\subsection{Proof of Example 2.4}

It follows from [12, Lemma A.2] that there exists $\zeta \in \mathcal{H}^{2}\left(\mathbb{R}^{1 \times 1}\right)$ with

$$
\ln \mathcal{E}(\zeta \cdot W) \in \mathcal{S}^{\infty}(\mathbb{R}) \text { and } E\left[e^{\frac{\pi^{2}+1}{4} \int_{0}^{T} \zeta_{t} d W_{t}}\right]=\infty
$$

Now for any $t \in[0, T]$, we denote

$$
Y_{t}^{1}=-\int_{0}^{t}\left|\zeta_{s}\right|^{2} d s+\int_{0}^{t} \zeta_{s} d W_{s}, \quad Z_{t}^{1}=\zeta_{t} .
$$

One can verify that $\left(Y^{1}, Z^{1}\right)$ is the unique solution to the following BSDE

$$
Y_{t}^{1}=\xi^{1}+\int_{t}^{T} \frac{1}{2}\left|Z_{s}^{1}\right|^{2} d s-\int_{t}^{T} Z_{s}^{1} d W_{s}
$$

in $\mathcal{S}^{\infty}(\mathbb{R}) \times B M O$ with $\xi^{1}=-\int_{0}^{T}\left|\zeta_{s}\right|^{2} d s+\int_{0}^{T} \zeta_{s} d W_{s}$. Therefore we have

$$
e^{\frac{\pi^{2}+1}{8} \int_{0}^{T}\left|Z_{t}^{1}\right|^{2} d t}=e^{\frac{\pi^{2}+1}{4}\left(Y_{0}^{1}-\xi^{1}\right)+\frac{\pi^{2}+1}{4} \int_{0}^{T} Z_{t}^{1} d W_{t}}
$$

which implies that

$$
E\left[e^{\frac{\pi^{2}+1}{8} \int_{0}^{T}\left|Z_{t}^{1}\right|^{2} d t}\right]=\infty
$$

since $Y_{0}^{1}$ and $\xi^{1}$ are bounded. On the other hand, the second equation in BSDE (2.2) implies that

$$
E\left[e^{\frac{\pi^{2}+1}{8} \int_{0}^{T}\left|Z_{t}^{1}\right|^{2} d t}\right]=e^{Y_{0}^{2}} E\left[\mathcal{E}_{T}\left(Z^{2} \cdot W\right)\right] \leq e^{Y_{0}^{2}}
$$

since $\mathcal{E}\left(Z^{2} \cdot W\right)$ is a positive supermartingale. Hence by setting

$$
\xi^{1}=-\int_{0}^{T}\left|\zeta_{s}\right|^{2} d s+\int_{0}^{T} \zeta_{s} d W_{s} \in L^{\infty}\left(\mathcal{F}_{T}\right)
$$

we have $Y_{0}^{2}=\infty$ which concludes the proof. 
A type of globally solvable BSDEs with triangularly quadratic generators

\section{Triangularly quadratic BSDEs with path dependence in value process}

Based on the arguments in the above section, we are able to consider the following type of triangularly quadratic BSDEs with path dependence in value process:

$$
\left\{\begin{aligned}
Y_{t}^{1}= & \xi^{1}+\int_{t}^{T}\left(\frac{1}{2}\left|Z_{s}^{1}\right|^{2}+Z_{s}^{1} l^{1}\left(s, G_{s}^{1}(Y)\right)+h^{1}\left(s, G_{s}^{1}(Y), Z_{s}\right)\right) d s-\int_{t}^{T} Z_{s}^{1} d W_{s}, \\
Y_{t}^{i}= & \xi^{i}+\int_{t}^{T}\left(\frac{1}{2}\left|Z_{s}^{i}\right|^{2}+Z_{s}^{i} l^{i}\left(s, G_{s}^{i}(Y), Z_{s}\right)-k^{i}\left(s, Z_{s}\right)+h^{i}\left(s, G_{s}^{i}(Y), Z_{s}\right)\right) d s \\
& -\int_{t}^{T} Z_{s}^{i} d W_{s}, \quad i=2, \ldots, n,
\end{aligned}\right.
$$

where $\xi$ is $\mathbb{R}^{d}$-valued and $\mathcal{F}_{T}$-measurable random variable which is bounded and for any $0 \leq t \leq T, G_{t}^{i}: \mathcal{C}_{T}\left(\mathbb{R}^{n}\right) \rightarrow \mathbb{R}^{n}$ is a function for $i=1, \ldots, n$. We suppose that the sequence of functions $G^{i}, i=1, \ldots, n$ satisfy the following assumption.

(A5) For any $0 \leq t \leq T$ and $y, \bar{y} \in \mathcal{C}_{T}\left(\mathbb{R}^{n}\right)$, we have $G_{t}^{i}(0)=0$ and

$$
\begin{aligned}
& G_{t}^{i}(y)=G_{t}^{i}\left(\left\{y_{s \wedge t}\right\}_{0 \leq s \leq t \leq T}\right) \\
& \left|G_{t}^{i}(y)-G_{t}^{i}(\bar{y})\right| \leq \sup _{0 \leq u \leq t}\left|y_{u}-\bar{y}_{u}\right|
\end{aligned}
$$

Remark 4.1. Given some $\epsilon \geq 0$, typical examples satisfying assumption (A5) are

- the delayed value of the solution, $G_{t}^{i}: y \mapsto y_{(t-\epsilon)^{+}}$;

- the recent maximum of the solution, $G_{t}^{i}: y \mapsto \sup _{(t-\epsilon)+\leq u \leq t} y_{u}$;

- the averaged recent value of the solution, $G_{t}^{i}: y \mapsto \frac{1}{\epsilon} \int_{(t-\epsilon)}^{t} y_{u} d u$;

- the cumulated recent value of the solution, $G_{t}^{i}: y \mapsto \int_{(t-\epsilon)}^{t} y_{u} d u$ for $\epsilon \leq 1$ or $T \leq 1$.

Theorem 4.2. Assume (A1)-(A5) hold, then there exist constants $T_{\eta}, C_{1}$ and $C_{2}$ only depending on $\alpha, C$ and $\|\xi\|_{\infty}$ such that for $T \leq T_{\eta}$, BSDE (4.1) admits a unique solution $(Y, Z)$ such that $(Y, Z \cdot W) \in \mathcal{S}^{\infty}\left(\mathbb{R}^{n}\right) \times B M O$ with $\|Y\|_{\mathcal{S}^{\infty}} \leq C_{1}$ and $\|Z \cdot W\|_{B M O} \leq C_{2}$.

Proof. Under the assumptions (A1)-(A5), it follows similarly from Step 1, Step 2 and Step 3 in the proof of Theorem 2.2 that there exist $T_{\bar{\eta}}, C_{1}$ and $C_{2}$ only depending on $\alpha$, $C$ and $\|\xi\|_{\infty}$ such that for any $(y, z \cdot W) \in \mathcal{S}^{\infty}\left(\mathbb{R}^{n}\right) \times B M O$ such that $\|y\|_{\mathcal{S}^{\infty}} \leq C_{1}$ and $\|z \cdot W\|_{B M O} \leq C_{2}$, the following BSDE

$$
\left\{\begin{aligned}
Y_{t}^{1}= & \xi^{1}+\int_{t}^{T}\left(\frac{1}{2}\left|Z_{s}^{1}\right|^{2}+Z_{s}^{1} l^{1}\left(s, G_{s}^{1}(y)\right)+h^{1}\left(s, G_{s}^{1}(y), z_{s}\right)\right) d s-\int_{t}^{T} Z_{s}^{1} d W_{s} \\
Y_{t}^{i}= & \xi^{i}+\int_{t}^{T}\left(\frac{1}{2}\left|Z_{s}^{i}\right|^{2}+Z_{s}^{i} l^{i}\left(s, G_{s}^{i}(y), z_{s}\right)-k^{i}\left(s, Z_{s}\right)+h^{i}\left(s, G_{s}^{i}(y), z_{s}\right)\right) d s \\
& -\int_{t}^{T} Z_{s}^{i} d W_{s}, \quad i=2, \ldots, n
\end{aligned}\right.
$$

admits a unique solution $(Y, Z)$ such that $(Y, Z \cdot W) \in \mathcal{S}^{\infty}\left(\mathbb{R}^{n}\right) \times B M O$ with $\|Y\|_{\mathcal{S} \infty} \leq C_{1}$ and $\|Z \cdot W\|_{B M O} \leq C_{2}$. Moreover, it follows from Step 4 in the proof of Theorem 2.2 that there exist $T_{\eta} \leq T_{\bar{\eta}}$ only depending on $\alpha, C$ and $\|\xi\|_{\infty}$ such that we can define a contraction mapping $\varphi$ on the set

$$
\mathcal{M}=\left\{(y, z \cdot W) \in \mathcal{S}^{\infty}\left(\mathbb{R}^{n}\right) \times B M O:\|y\|_{\mathcal{S}^{\infty}} \leq C_{1} \text { and }\|z \cdot W\|_{B M O} \leq C_{2}\right\}
$$

by $\varphi(y, z):=(Y, Z)$, which concludes the proof. 
A type of globally solvable BSDEs with triangularly quadratic generators

Remark 4.3. For $T \leq 1$ and $G_{t}^{i}(y)=\int_{0}^{t} y_{s} d s$ for $i=1, \ldots, n$, BSDE (4.1) is equivalent to the following FBSDE

$$
\left\{\begin{aligned}
X_{t}= & \int_{0}^{t} Y_{s} d s \\
Y_{t}^{1}= & \xi^{1}+\int_{t}^{T}\left(\frac{1}{2}\left|Z_{s}^{1}\right|^{2}+Z_{s}^{1} l^{1}\left(s, X_{s}\right)+h^{1}\left(s, X_{s}, Z_{s}\right)\right) d s-\int_{t}^{T} Z_{s}^{1} d W_{s}, \\
Y_{t}^{i}= & \xi^{i}+\int_{t}^{T}\left(\frac{1}{2}\left|Z_{s}^{i}\right|^{2}+Z_{s}^{i} l^{i}\left(s, X_{s}, Z_{s}\right)-k^{i}\left(s, Z_{s}\right)+h^{i}\left(s, X_{s}, Z_{s}\right)\right) d s \\
& -\int_{t}^{T} Z_{s}^{i} d W_{s}, \quad i=2, \ldots, n,
\end{aligned}\right.
$$

which in general admits a solution only for a small time horizon. We refer to [21, 22] for related studies.

For a given delay parameter $\epsilon>0$, we consider the following BSDE with delay in value process:

$$
Y_{t}^{i}=\xi^{i}+\int_{t}^{T}\left(\frac{1}{2}\left|Z_{s}^{i}\right|^{2}+Z_{s}^{i} l^{i}\left(s, G_{s}^{i, \epsilon}(Y)\right)+h^{i}\left(s, G_{s}^{i, \epsilon}(Y), Z_{s}\right)\right) d s-\int_{t}^{T} Z_{s}^{i} d W_{s},
$$

where $\xi$ is $\mathbb{R}^{d}$-valued and $\mathcal{F}_{T}$-measurable random variable which is bounded and for any $0 \leq t \leq T, G_{t}^{i, \epsilon}: \mathcal{C}_{T}\left(\mathbb{R}^{n}\right) \rightarrow \mathbb{R}^{n}$ is a function for $i=1, \ldots, n$. We suppose that the sequence of functions $G^{i, \epsilon}, i=1, \ldots, n$ satisfy the following assumption.

(A6) For any $0 \leq t \leq T, \epsilon>0$ and $y, \bar{y} \in \mathcal{C}_{T}\left(\mathbb{R}^{n}\right)$, we have $G_{t}^{i, \epsilon}(0)=0$ and

$$
\begin{aligned}
& G_{t}^{i, \epsilon}(y)=G_{t}^{i, \epsilon}\left(\left\{y_{s \wedge t}\right\}_{0 \leq s \leq t \leq T}\right) ; \\
& \left|G_{t}^{i, \epsilon}(y)-G_{t}^{i, \epsilon}(\bar{y})\right| \leq \sup _{(t-\epsilon)^{+} \leq u \leq t}\left|y_{u}-\bar{y}_{u}\right| .
\end{aligned}
$$

We also make the following assumptions:

(A7) For $i=1, \ldots, n, l^{i}: \Omega \times[0, T] \times \mathbb{R}^{n} \rightarrow \mathbb{R}$ satisfies that $l^{i}(\cdot, y)$ is adapted for each $y \in \mathbb{R}^{n}$. Moreover, it holds that

$$
\begin{aligned}
& \left|l^{i}(t, y)\right| \leq C, \quad y \in \mathbb{R}^{n} \\
& \left|l^{i}(t, y)-l^{i}(t, \bar{y})\right| \leq C|y-\bar{y}|, \quad y, \bar{y} \in \mathbb{R}^{n}
\end{aligned}
$$

(A8) $h: \Omega \times[0, T] \times \mathbb{R}^{n} \times \mathbb{R}^{n \times d} \rightarrow \mathbb{R}^{n}$ satisfies that $h(\cdot, y, z)$ is adapted for each $y \in \mathbb{R}^{n}$ and $z \in \mathbb{R}^{n \times d}$. Moreover, there exists $\alpha \in[-1,1)$ such that

$$
\begin{aligned}
& -C\left(1+|z|^{1+\alpha}\right) \leq h(t, y, z) \leq 0, \quad y \in \mathbb{R}^{n}, z \in \mathbb{R}^{n \times d} ; \\
& |h(t, y, z)-h(t, \bar{y}, \bar{z})| \leq C|y-\bar{y}|+C\left(1+|z|^{\alpha^{+}}+|\bar{z}|^{\alpha^{+}}\right)|z-\bar{z}|,
\end{aligned}
$$

for $y, \bar{y} \in \mathbb{R}^{n}$ and $z, \bar{z} \in \mathbb{R}^{n \times d}$.

We denote

$$
\begin{aligned}
\bar{\beta} & =n+n\left(1+4 n^{2}\left(1+\left(4 C e^{\|\xi\|_{\infty}}\right)^{n}\right)\right)\left(e^{\|\xi\|_{\infty}}+\|\xi\|_{\infty}+\left(C^{2} e^{2\|\xi\|_{\infty}}+2 C e^{\|\xi\|_{\infty}}\right) T\right) \\
& +\frac{1-\alpha}{8(1-\alpha)}\left(4(1+\alpha) n C e^{\|\xi\|_{\infty}}\left(1+4 n^{2}\left(1+\left(4 C e^{\|\xi\|_{\infty}}\right)^{n}\right)\right)\right)^{\frac{2}{1-\alpha}} T, \\
\tilde{\Delta} & =\Delta(C \sqrt{2 T}+16 \sqrt{2} \bar{\beta}), \\
\tilde{\delta} & =\delta(C \sqrt{2 T}+16 \sqrt{2} \bar{\beta}), \\
\epsilon_{0} & =\frac{\tilde{\delta}}{4 n C^{2}(2 e \tilde{\delta} T+2 e \tilde{\Delta} \tilde{\delta} \bar{\beta}+1)} .
\end{aligned}
$$


A type of globally solvable BSDEs with triangularly quadratic generators

Theorem 4.4. Assume (A6)-(A8) hold. Then for any $0<\epsilon \leq \epsilon_{0}$, BSDE (4.2) admits a unique solution $(Y, Z)$ such that $(Y, Z \cdot W) \in \mathcal{S}^{\infty}\left(\mathbb{R}^{n}\right) \times B M O$.

Proof. For $y \in \mathcal{S}^{\infty}\left(\mathbb{R}^{n}\right)$, if follows from Theorem 2.3 that the following BSDE

$$
Y_{t}^{i}=\xi^{i}+\int_{t}^{T}\left(\frac{1}{2}\left|Z_{s}^{i}\right|^{2}+Z_{s}^{i} l^{i}\left(s, G_{s}^{i, \epsilon}(y)\right)+h^{i}\left(s, G_{s}^{i, \epsilon}(y), Z_{s}\right)\right) d s-\int_{t}^{T} Z_{s}^{i} d W_{s}
$$

admits a unique solution $(Y, Z)$ such that $(Y, Z \cdot W) \in \mathcal{S}^{\infty}\left(\mathbb{R}^{n}\right) \times B M O$ and

$$
\|Y\|_{\mathcal{S}^{\infty}} \leq \bar{\beta}, \quad\|Z \cdot W\|_{B M O}^{2} \leq 8 \bar{\beta} .
$$

Let us introduce the function $\varphi: \mathcal{S}^{\infty}\left(\mathbb{R}^{n}\right) \mapsto \mathcal{S}^{\infty}\left(\mathbb{R}^{n}\right)$ such that for any $y \in \mathcal{S}^{\infty}\left(\mathbb{R}^{n}\right)$, $\varphi(y)=Y$ is the first component to the following BSDE

$$
Y_{t}^{i}=\xi^{i}+\int_{t}^{T}\left(\frac{1}{2}\left|Z_{s}^{i}\right|^{2}+Z_{s}^{i} l^{i}\left(s, G_{s}^{i, \epsilon}(y)\right)+h^{i}\left(s, G_{s}^{i, \epsilon}(y), Z_{s}\right)\right) d s-\int_{t}^{T} Z_{s}^{i} d W_{s}
$$

We consider $(y, \bar{y}) \in \mathcal{S}^{\infty}\left(\mathbb{R}^{n}\right) \times \mathcal{S}^{\infty}\left(\mathbb{R}^{n}\right)$ and denote by $(Y, Z)$ and $(\bar{Y}, \bar{Z})$ the solutions to the associated BSDEs. From the above, we have

$$
\|Z \cdot W\|_{B M O}^{2} \leq 8 \bar{\beta}, \quad\|\bar{Z} \cdot W\|_{B M O}^{2} \leq 8 \bar{\beta} .
$$

Moreover, applying Itô's formula to $e^{\gamma t}\left|Y_{t}^{i}-\bar{Y}_{t}^{i}\right|^{2}$, we have

$$
\begin{aligned}
e^{\gamma t}\left|Y_{t}^{i}-\bar{Y}_{t}^{i}\right|^{2}+\int_{t}^{T} e^{\gamma s}\left|Z_{s}^{i}-\bar{Z}_{s}^{i}\right|^{2} d s \\
=2 \int_{t}^{T} e^{\gamma s}\left(Y_{s}^{i}-\bar{Y}_{s}^{i}\right)\left(\frac{1}{2}\left|Z_{s}^{i}\right|^{2}-\frac{1}{2}\left|\bar{Z}_{s}^{i}\right|^{2}+Z_{s}^{i} l^{i}\left(s, G_{s}^{i, \epsilon}(y)\right)-\bar{Z}_{s}^{i} l^{i}\left(s, G_{s}^{i, \epsilon}(\bar{y})\right)\right) d s \\
\quad+2 \int_{t}^{T} e^{\gamma s}\left(Y_{s}^{i}-\bar{Y}_{s}^{i}\right)\left(h^{i}\left(s, G_{s}^{i, \epsilon}(y), Z_{s}\right)-h^{i}\left(s, G_{s}^{i, \epsilon}(\bar{y}), \bar{Z}_{s}\right)\right) d s \\
\quad-\gamma \int_{t}^{T} e^{\gamma s}\left|Y_{s}^{i}-\bar{Y}_{s}^{i}\right|^{2} d s-2 \int_{t}^{T} e^{\gamma s}\left(Y_{s}^{i}-\bar{Y}_{s}^{i}\right)\left(Z_{s}^{i}-\bar{Z}_{s}^{i}\right) d W_{s} \\
=2 \int_{t}^{T} e^{\gamma s}\left(Y_{s}^{i}-\bar{Y}_{s}^{i}\right) \bar{Z}_{s}^{i}\left(l^{i}\left(s, G_{s}^{i, \epsilon}(y)\right)-l^{i}\left(s, G_{s}^{i, \epsilon}(\bar{y})\right)\right) d s \\
\quad+2 \int_{t}^{T} e^{\gamma s}\left(Y_{s}^{i}-\bar{Y}_{s}^{i}\right)\left(h^{i}\left(s, G_{s}^{i, \epsilon}(y), Z_{s}\right)-h^{i}\left(s, G_{s}^{i, \epsilon}(\bar{y}), \bar{Z}_{s}\right)\right) d s \\
\quad-\gamma \int_{t}^{T} e^{\gamma s}\left|Y_{s}^{i}-\bar{Y}_{s}^{i}\right|^{2} d s-2 \int_{t}^{T} e^{\gamma s}\left(Y_{s}^{i}-\bar{Y}_{s}^{i}\right)\left(Z_{s}^{i}-\bar{Z}_{s}^{i}\right) d \bar{W}_{s}^{i},
\end{aligned}
$$

where $\bar{W}_{t}^{i}=W_{t}-\int_{0}^{t}\left(\frac{1}{2}\left(Z_{s}^{i}+\bar{Z}_{s}^{i}\right)+l^{i}\left(s, G_{s}^{i, \epsilon}(y)\right)\right) d s$ is a Brownian motion under the equivalent probability measure $\bar{P}^{i}$ defined by

$$
\frac{d \bar{P}^{i}}{d P}=\mathcal{E}_{T}\left(\left(\frac{1}{2}\left(Z_{.}^{i}+\bar{Z}_{.}^{i}\right)+l^{i}\left(\cdot, G^{i, \epsilon}(y)\right)\right) \cdot W\right) .
$$


A type of globally solvable BSDEs with triangularly quadratic generators

Therefore, we have

$$
\begin{aligned}
& e^{\gamma t}\left|Y_{t}^{i}-\bar{Y}_{t}^{i}\right|^{2}+\int_{t}^{T} e^{\gamma s}\left|Z_{s}^{i}-\bar{Z}_{s}^{i}\right|^{2} d s \\
& \leq 2 C \int_{t}^{T} e^{\gamma s}\left|Y_{s}^{i}-\bar{Y}_{s}^{i}\right|\left|\bar{Z}_{s}^{i}\right|\left|G_{s}^{i, \epsilon}(y)-G_{s}^{i, \epsilon}(\bar{y})\right| d s \\
& \quad+2 C \int_{t}^{T} e^{\gamma s}\left|Y_{s}^{i}-\bar{Y}_{s}^{i}\right|\left(\left|G_{s}^{i, \epsilon}(y)-G_{s}^{i, \epsilon}(\bar{y})\right|+\left|Z_{s}-\bar{Z}_{s}\right|\right) d s \\
& \quad-\gamma \int_{t}^{T} e^{\gamma s}\left|Y_{s}^{i}-\bar{Y}_{s}^{i}\right|^{2} d s-2 \int_{t}^{T} e^{\gamma s}\left(Y_{s}^{i}-\bar{Y}_{s}^{i}\right)\left(Z_{s}^{i}-\bar{Z}_{s}^{i}\right) d \bar{W}_{s}^{i} \\
& \leq\left(8 n C^{2} T e^{\gamma \epsilon}+\frac{4 n \tilde{\Delta} C^{2}}{\tilde{\delta}}+8 n C^{2} \tilde{\Delta} \bar{\beta} e^{\gamma \epsilon}\right) \int_{t}^{T} e^{\gamma s}\left|Y_{s}^{i}-\bar{Y}_{s}^{i}\right|^{2} d s-\gamma \int_{t}^{T} e^{\gamma s}\left|Y_{s}^{i}-\bar{Y}_{s}^{i}\right|^{2} d s \\
& \quad+\frac{1}{64 n \tilde{\Delta} \bar{\beta} e^{\gamma \epsilon}} \int_{t}^{T} e^{\gamma s}\left|\bar{Z}_{s}^{i}\right|^{2}\left|G_{s}^{i, \epsilon}(y)-G_{s}^{i, \epsilon}(\bar{y})\right|^{2} d s+\frac{1}{8 n T e^{\gamma \epsilon}} \int_{t}^{T} e^{\gamma s}\left|G_{s}^{i, \delta}(y)-G_{s}^{i, \delta}(\bar{y})\right|^{2} d s \\
& \quad+\frac{\tilde{\delta}}{4 n \tilde{\Delta}} \int_{t}^{T} e^{\gamma s}\left|Z_{s}-\bar{Z}_{s}\right|^{2} d s-2 \int_{t}^{T} e^{\gamma s}\left(Y_{s}^{i}-\bar{Y}_{s}^{i}\right)\left(Z_{s}^{i}-\bar{Z}_{s}^{i}\right) d \bar{W}_{s}^{i}
\end{aligned}
$$

where the last inequality comes from Young's inequality. Since $\epsilon \leq \epsilon_{0}$, by letting $\lambda=\frac{1}{\epsilon}$, we have

$$
\begin{aligned}
& e^{\gamma t}\left|Y_{t}^{i}-\bar{Y}_{t}^{i}\right|^{2}+\int_{t}^{T} e^{\gamma s}\left|Z_{s}^{i}-\bar{Z}_{s}^{i}\right|^{2} d s \\
& \leq \frac{1}{64 n \tilde{\Delta} \bar{\beta} e^{\gamma \epsilon}} \int_{t}^{T} e^{\gamma s}\left|\bar{Z}_{s}^{i}\right|^{2}\left|G_{s}^{i, \epsilon}(y)-G_{s}^{i, \epsilon}(\bar{y})\right|^{2} d s+\frac{1}{8 n T e^{\gamma \epsilon}} \int_{t}^{T} e^{\gamma s}\left|G_{s}^{i, \delta}(y)-G_{s}^{i, \delta}(\bar{y})\right|^{2} d s \\
& \quad+\frac{\tilde{\delta}}{4 n \tilde{\Delta}} \int_{t}^{T} e^{\gamma s}\left|Z_{s}-\bar{Z}_{s}\right|^{2} d s-2 \int_{t}^{T} e^{\gamma s}\left(Y_{s}^{i}-\bar{Y}_{s}^{i}\right)\left(Z_{s}^{i}-\bar{Z}_{s}^{i}\right) d \bar{W}_{s}^{i} .
\end{aligned}
$$

Noting that for $\epsilon>0$, we have

$$
\begin{aligned}
e^{\gamma s}\left|G_{s}^{i, \epsilon}(y)-G_{s}^{i, \epsilon}(\bar{y})\right|^{2} & \leq e^{\gamma s} \sup _{(s-\epsilon)^{+} \leq r \leq s}\left|y_{r}-\bar{y}_{r}\right|^{2} \\
& \leq e^{\gamma \epsilon} \sup _{(s-\epsilon)^{+} \leq r \leq s} e^{\gamma r}\left|y_{r}-\bar{y}_{r}\right|^{2} d s \\
& \leq e^{\gamma \epsilon}|| e^{\gamma \cdot}|y-\bar{y}|^{2} \|_{\mathcal{S}^{\infty}} .
\end{aligned}
$$

Hence we have

$$
\begin{aligned}
& e^{\gamma t}\left|Y_{t}^{i}-\bar{Y}_{t}^{i}\right|^{2}+\int_{t}^{T} e^{\gamma s}\left|Z_{s}^{i}-\bar{Z}_{s}^{i}\right|^{2} d s
\end{aligned}
$$

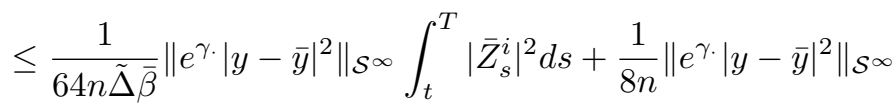

$$
\begin{aligned}
& +\frac{\tilde{\delta}}{4 n \tilde{\Delta}} \int_{t}^{T} e^{\gamma s}\left|Z_{s}-\bar{Z}_{s}\right|^{2} d s-2 \int_{t}^{T} e^{\gamma s}\left(Y_{s}^{i}-\bar{Y}_{s}^{i}\right)\left(Z_{s}^{i}-\bar{Z}_{s}^{i}\right) d \bar{W}_{s}^{i} .
\end{aligned}
$$

Therefore, it follows from Lemma 3.1 that

$$
\begin{aligned}
& \left\|e^{\gamma \cdot}\left|Y^{i}-\bar{Y}^{i}\right|^{2}\right\|_{\mathcal{S}^{\infty}}+\tilde{\delta}\left\|e^{\frac{\gamma \cdot}{2}}\left(Z^{i}-\bar{Z}^{i}\right) \cdot W\right\|_{B M O}^{2}
\end{aligned}
$$

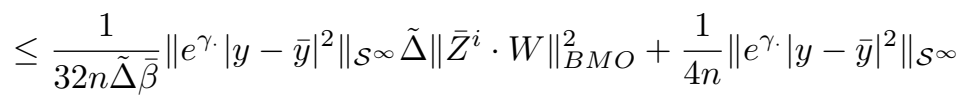

$$
\begin{aligned}
& +\frac{\tilde{\delta}}{2 n \tilde{\Delta}} \tilde{\Delta}\left\|e^{\frac{\gamma \cdot}{2}}(Z-\bar{Z}) \cdot W\right\|_{B M O}^{2} \\
& \leq \frac{1}{2 n}\left\|e^{\gamma \cdot}|y-\bar{y}|^{2}\right\|_{\mathcal{S}^{\infty}}+\frac{\tilde{\delta}}{2 n}\left\|e^{\frac{\gamma \cdot}{2}}(Z-\bar{Z}) \cdot W\right\|_{B M O}^{2} .
\end{aligned}
$$


A type of globally solvable BSDEs with triangularly quadratic generators

Finally, we deduce that

$$
\left\|e^{\gamma \cdot}|Y-\bar{Y}|^{2}\right\|_{\mathcal{S}^{\infty}} \leq \frac{1}{2}\left\|e^{\gamma \cdot}|y-\bar{y}|^{2}\right\|_{\mathcal{S}^{\infty}} .
$$

Thus, $\varphi$ is a contraction on $\mathcal{S}^{\infty}\left(\mathbb{R}^{n}\right)$ so that there exists a unique solution $(Y, Z)$ to $\operatorname{BSDE}$ (4.2) such that $(Y, Z \cdot W) \in \mathcal{S}^{\infty}\left(\mathbb{R}^{n}\right) \times B M O$.

\section{References}

[1] J.M. Bismut. Conjugate convex functions in optimal stochastic control. J. Math. Anal. Appl. 44, (1973), 384-404. MR-0329726

[2] K. Bahlali, E. H. Essaky, M. Hassani. Multidimensional BSDEs with super-linear growth coefficient: Application to degenerate systems of semilinear PDEs. C.R. Acad. Sci. Paris Sér. I Math. 348, (2010), 677-682. MR-2652497

[3] P. Briand and R. Elie. A simple constructive approach to quadratic BSDEs with or without delay. Stochastic Processes and their Applications. 123(8), 2013, 2921-2939. MR-3062430

[4] P. Briand and Y. Hu. BSDE with quadratic growth and unbounded terminal value. Probability Theory and Related Fields. 136, (2006), 604-618. MR-2257138

[5] P. Briand and Y. Hu. Quadratic BSDEs with convex generators and unbounded terminal conditions. Probability Theory and Related Fields. 141, (2008), 543-567. MR-2391164

[6] P. Cheridito and K. Nam. BSDEs with terminal conditions that have bounded Malliavin derivative. Journal of Functional Analysis 266, (2014), 1257-1285. MR-3146818

[7] P. Cheridito and K. Nam. Multidimensional quadratic and subquadratic BSDEs with special structure. Stochastics 87, (2015), 871-884. MR-3390237

[8] B. Chikvinidze and M. Mania. New proofs of some results on bounded mean oscillation martingales using Backward stochastic differential equations. J. Theor. Probab. 27, (2014), 1213-1228. MR-3278938

[9] F. Delbaen, Y. Hu, X. Bao. Backward SDEs with superquadratic growth. Probability Theory and Related Fields. 150, (2011), 145-192. MR-2800907

[10] N. El Karoui and S. Hamadène. BSDEs and risk-sensitive control, zero-sum and non-zero sum game problems of stochastic functional differential equations. Stochastic Process. Appl. 107, (2003), 145-169. MR-1995925

[11] C. Frei. Splitting multidimensional BSDEs and finding local equilibria. Stochastic Process. Appl. 124, (2014), 2654-2671. MR-3200729

[12] C. Frei and G. dos Reis. A financial market with interacting investors: does an equilibrium exist? Math. Financ. Econ. 4, (2011), 161-182. MR-2796281

[13] J. Harter and A. Richou. A stability approach for solving multidimensional quadaratic BSDEs. Electron. J. Probab. 24(4), 2019, 1-51. MR-3916324

[14] Y. Hu and S. Tang. Multi-dimensional backward stochastic differential equations of diagonally quadratic generators. Stochastic Process. Appl. 126, (2016), 1066-1086. MR-3461191

[15] Y. Hu and S. Tang. Non-zero sum quadratic differential game of BSDEs and multi-dimensional diagonally quadratic BSDE. IFAC-PapersOnline 49, (2016), 308-309.

[16] A. Jamneshan, M. Kupper and P. Luo. Multidimensional quadratic BSDEs with separated generators. Electronic Communications in Probability. 22(58), 2017, 1-10. MR-3718708

[17] C. Kardaras, H. Xing and G. Žitković. Incomplete stochastic equilibria with exponential utilities close to Pareto optimality, arXiv:1505.07224v1.

[18] N. Kazamaki. Continuous Exponential Martingale and BMO. Lecture Notes in Mathematics, vol. 1579. Springer-Verlag, Berlin, 1994. viii+91 pp. MR-1299529

[19] M. Kobylanski. Backward stochastic differential equations and partial differential equations with quadratic growth. Annals of Probability. 28, (2000), 558-602. MR-1782267

[20] D. Kramkov and S. Pulido. A system of quadratic BSDEs arising in a price impact model. Ann. Appl. Probab. 26, (2016), 794-817. MR-3476625 
A type of globally solvable BSDEs with triangularly quadratic generators

[21] M. Kupper, P. Luo and L. Tangpi. Multidimensional Markovian FBSDEs with super-quadratic growth. Stochastic Processes and their Applications. 129(3), 2019, 902-923. MR-3913273

[22] P. Luo and L. Tangpi. Solvability of coupled FBSDEs with diagonally quadratic generators. Stochastics and Dynamics. 17(6), (2017), 1750043. MR-3685636

[23] F. Masiero and A. Richou. A note on the existence of solutions to Markovian superquadratic BSDEs with an unbounded ternimal condtion. Electron. J. Probab 18, (2013), 1-15. MR3048122

[24] E.Pardoux and S. G. Peng. Adapted solution of a backward stochastic differential equation. System Control Lett. 14, (1990), 55-61. MR-1037747

[25] A. Richou. Markovian quadratic and superquadratic BSDEs with an unbounded terminal condition. Stochastic Process. Appl. 122, (2012), 3173-3208. MR-2946439

[26] R. Tevzadze. Solvability of backward stochastic differential equations with quadratic growth. Stochastic Process. Appl. 118, (2008), 503-515. MR-2389055

[27] N. Touzi. Optimal Stochastic Control, Stochastic Target Problems, and Backward SDE. Fields Institute Monographs, vol. 29. Springer, New York, 2013. x+214 pp. MR-2976505

[28] H. Xing and G. Žitković. A class of globally solvable Markovian quadratic BSDE systems and applications. Ann. Probab. 46(1), 2018, 491-550. MR-3758736

Acknowledgments. We would like to thank the editor and one referee for their careful reading and invaluable suggestions. 


\section{Electronic Journal of Probability Electronic Communications in Probability}

\section{Advantages of publishing in EJP-ECP}

- Very high standards

- Free for authors, free for readers

- Quick publication (no backlog)

- Secure publication $\left(\mathrm{LOCKSS}^{1}\right)$

- Easy interface (EJMS²)

\section{Economical model of EJP-ECP}

- Non profit, sponsored by $\mathrm{IMS}^{3}, \mathrm{BS}^{4}$, ProjectEuclid ${ }^{5}$

- Purely electronic

\section{Help keep the journal free and vigorous}

- Donate to the IMS open access fund ${ }^{6}$ (click here to donate!)

- Submit your best articles to EJP-ECP

- Choose EJP-ECP over for-profit journals

\footnotetext{
${ }^{1}$ LOCKSS: Lots of Copies Keep Stuff Safe http://www. lockss.org/

${ }^{2}$ EJMS: Electronic Journal Management System http://www.vtex.lt/en/ejms.html

${ }^{3}$ IMS: Institute of Mathematical Statistics http://www.imstat.org/

${ }^{4}$ BS: Bernoulli Society http://www. bernoulli-society.org/

${ }^{5}$ Project Euclid: https://projecteuclid.org/

${ }^{6}$ IMS Open Access Fund: http://www.imstat.org/publications/open.htm
} 